\title{
SEVEN-YEAR WILKINSON MICROWAVE ANISOTROPY PROBE (WMAP*) OBSERVATIONS: SKY MAPS, SYSTEMATIC ERRORS, AND BASIC RESULTS
}

\author{
N. JarosiK ${ }^{1}$, C. L. BennetT ${ }^{2}$, J. Dunkley ${ }^{3}$, B. Gold ${ }^{2}$, M. R. Greason ${ }^{4}$, M. HalPern ${ }^{5}$, R. S. Hill ${ }^{4}$, G. HinshaW ${ }^{6}$, A. Kogut ${ }^{6}$,

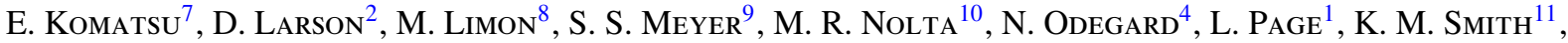 \\ D. N. Spergel ${ }^{11,12}$, G. S. Tucker ${ }^{13}$, J. L. Weiland ${ }^{4}$, E. Wollack ${ }^{6}$, and E. L. Wright ${ }^{14}$ \\ ${ }^{1}$ Department of Physics, Jadwin Hall, Princeton University, Princeton, NJ 08544-0708, USA \\ ${ }^{2}$ Department of Physics \& Astronomy, The Johns Hopkins University, 3400 North Charles Street, Baltimore, MD 21218-2686, USA \\ ${ }^{3}$ Department of Astrophysics, University of Oxford, Keble Road, Oxford OX1 3RH, UK \\ ${ }^{4}$ ADNET Systems, Inc., 7515 Mission Dr., Suite A100 Lanham, MD 20706, USA \\ ${ }^{5}$ Department of Physics and Astronomy, University of British Columbia, Vancouver, BC V6T 1Z1, Canada \\ ${ }^{6}$ Code 665, NASA/Goddard Space Flight Center, Greenbelt, MD 20771, USA \\ ${ }^{7}$ Department of Astronomy, University of Texas, Austin, 2511 Speedway, RLM 15.306, Austin, TX 78712, USA \\ ${ }^{8}$ Columbia Astrophysics Laboratory, 550 West 120th Street, Mail Code 5247, New York, NY 10027-6902, USA \\ ${ }_{9}^{9}$ Departments of Astrophysics and Physics, KICP and EFI, University of Chicago, Chicago, IL 60637, USA \\ ${ }^{10}$ Canadian Institute for Theoretical Astrophysics, 60 St. George Street, University of Toronto, Toronto, ON M5S 3H8, Canada \\ ${ }^{11}$ Department of Astrophysical Sciences, Peyton Hall, Princeton University, Princeton, NJ 08544-1001, USA \\ ${ }_{12}$ Princeton Center for Theoretical Physics, Princeton University, Princeton, NJ 08544, USA \\ ${ }^{13}$ Department of Physics, Brown University, 182 Hope St., Providence, RI 02912-1843, USA \\ ${ }^{14}$ UCLA Physics \& Astronomy, P.O. Box 951547, Los Angeles, CA 90095-1547, USA \\ Received 2010 January 25; accepted 2010 March 9; published 2011 January 11
}

\begin{abstract}
New full-sky temperature and polarization maps based on seven years of data from WMAP are presented. The new results are consistent with previous results, but have improved due to reduced noise from the additional integration time, improved knowledge of the instrument performance, and improved data analysis procedures. The improvements are described in detail. The seven-year data set is well fit by a minimal six-parameter flat $\Lambda$ CDM model. The parameters for this model, using the WMAP data in conjunction with baryon acoustic oscillation data from the Sloan Digital Sky Survey and priors on $H_{0}$ from Hubble Space Telescope observations, are $\Omega_{b} h^{2}=$ $0.02260 \pm 0.00053, \Omega_{c} h^{2}=0.1123 \pm 0.0035, \Omega_{\Lambda}=0.728_{-0.016}^{+0.015}, n_{s}=0.963 \pm 0.012, \tau=0.087 \pm 0.014$, and $\sigma_{8}=0.809 \pm 0.024$ (68\% CL uncertainties). The temperature power spectrum signal-to-noise ratio per multipole is greater that unity for multipoles $\ell \lesssim 919$, allowing a robust measurement of the third acoustic peak. This measurement results in improved constraints on the matter density, $\Omega_{m} h^{2}=0.1334_{-0.0055}^{+0.0056}$, and the epoch of matter-radiation equality, $z_{\mathrm{eq}}=3196_{-133}^{+134}$, using WMAP data alone. The new WMAP data, when combined with smaller angular scale microwave background anisotropy data, result in a $3 \sigma$ detection of the abundance of primordial helium, $Y_{\mathrm{He}}=0.326 \pm 0.075$. When combined with additional external data sets, the WMAP data also yield better determinations of the total mass of neutrinos, $\sum m_{v} \leqslant 0.58 \mathrm{eV}$ (95\% CL), and the effective number of neutrino species, $N_{\text {eff }}=4.34_{-0.88}^{+0.86}$. The power-law index of the primordial power spectrum is now determined to be $n_{s}=$ $0.963 \pm 0.012$, excluding the Harrison-Zel'dovich-Peebles spectrum by $>3 \sigma$. These new WMAP measurements provide important tests of big bang cosmology.
\end{abstract}

Key words: cosmic background radiation - space vehicles: instruments

\section{INTRODUCTION}

The Wilkinson Microwave Anisotropy Probe (WMAP) is a NASA sponsored satellite designed to map the cosmic microwave background (CMB) radiation over the entire sky in five frequency bands. It was launched in 2001 June from Kennedy Space Flight Center and began surveying the sky from its orbit around the Earth-Sun L2 point in 2001 August. This work and the accompanying papers comprise the fourth in a series of biennial data releases and incorporates seven years of observational data.

Results from the one-year, three-year, and five-year observations are summarized in Bennett et al. (2003a), Jarosik et al. (2007), and Hinshaw et al. (2009), respectively, and references therein. An overall description of the mission including instrument nomenclature is contained in Bennett et al. (2003b) and

\footnotetext{
* WMAP is the result of a partnership between Princeton University and NASA's Goddard Space Flight Center. Scientific guidance is provided by the WMAP Science Team.
}

Limon et al. (2010), while details of the optical system and radiometers can be found in Page et al. (2003b) and Jarosik et al. (2003).

The primary data product of WMAP are sets of calibrated sky maps at five frequency bands centered at $23 \mathrm{GHz}$ (K band), $33 \mathrm{GHz}$ (Ka band), $41 \mathrm{GHz}$ (Q band), $61 \mathrm{GHz}$ (V band), and $94 \mathrm{GHz}$ (W band), including measured noise levels and beam transfer functions that describe the smoothing of the sky signal resulting from the beam geometries. These maps are provided for Stokes $I, Q$, and $U$ parameters on a year-byyear basis and in a year co-added format, and at several pixel resolutions appropriate for various analyses. Changes relative to the previous data release include the inclusion of seven years of observational data, a new masking procedure that simplifies the map-making process, and improvements of the beam maps and window functions. Details of the processing used to generate these products are described in the remainder of this work.

In an accompanying paper, Gold et al. (2011) utilize the maps in the five frequency bands and some external data sets to 
Table 1

Noise and Calibration Summary for the Template Cleaned and Uncleaned Maps

\begin{tabular}{|c|c|c|c|c|}
\hline \multirow[t]{3}{*}{$\mathrm{DA}$} & $\sigma_{0}(I)$ & $\sigma_{0}(Q, U)$ & \multirow{3}{*}{$\begin{array}{c}\sigma_{0}(Q, U) \\
\text { Template Cleaned }^{\mathrm{b}} \\
(\mathrm{mK})\end{array}$} & \multirow{3}{*}{$\begin{array}{c}\Delta G / G^{\mathrm{a}} \\
(\%)\end{array}$} \\
\hline & \multicolumn{2}{|c|}{ Uncleaned } & & \\
\hline & $(\mathrm{mK})$ & $(\mathrm{mK})$ & & \\
\hline K1 & 1.437 & 1.456 & NA & -0.14 \\
\hline $\mathrm{Ka} 1$ & 1.470 & 1.490 & 2.192 & -0.01 \\
\hline Q1 & 2.254 & 2.280 & 2.741 & 0.01 \\
\hline Q2 & 2.140 & 2.164 & 2.602 & 0.01 \\
\hline V1 & 3.319 & 3.348 & 3.567 & -0.03 \\
\hline $\mathrm{V} 2$ & 2.955 & 2.979 & 3.174 & -0.03 \\
\hline W1 & 5.906 & 5.940 & 6.195 & -0.05 \\
\hline W2 & 6.572 & 6.612 & 6.896 & -0.04 \\
\hline W3 & 6.941 & 6.983 & 7.283 & -0.08 \\
\hline W4 & 6.778 & 6.840 & 7.134 & -0.12 \\
\hline
\end{tabular}

Notes.

a $\Delta G / G$ is the change in calibration of the current (seven-year) processing relative to the five-year processing. A positive value means that features in the seven-year maps are larger than the same features in the five-year map.

b The $\sigma_{0}$ value for the Stokes $I$ template cleaned maps is the same as for the uncleaned maps.

estimate levels of Galactic emission in each map, and describe the generation of a set of reduced foreground sky maps based on template cleaning, and a map generated using an Internal Linear Combination (ILC) of WMAP data, both of which are used for analysis of the CMB anisotropy signal.

Larson et al. (2011) describe the measurement of the angular power spectrum of the CMB obtained from the reduced foreground sky maps and the cosmological parameters obtained by fitting the CMB power spectra to current cosmological models.

The cosmological implications of the data, including the use of external data sets, are discussed by Komatsu et al. (2011), while Bennett et al. (2011) discuss a number of arguably anomalous results detected in previous WMAP data releases.

Weiland et al. (2011) describe the characteristics of a group of point-like objects observed by WMAP in the context of their use as microwave calibration sources for astronomical observations.

The remainder of this paper is organized as follows. Section 2 presents updates on the data processing procedures used to generate the seven-year sky maps and related data products. Section 3 describes ongoing efforts to characterize the WMAP beam shapes, while Section 4 presents the seven-year sky map data and power spectra, describes some additional analyses on the low- $\ell$ polarization power spectra, and summarizes the scientific results obtained from the latest WMAP data set.

\section{DATA PROCESSING UPDATES}

\subsection{Operations}

The sixth and seventh years of WMAP's operation span the interval from 2006 August 9, 00:00:00 UT (day number 222) to 2008 August 10, 00:00:00 UT (day number 222) and include nine short periods when observations were interrupted. These periods include eight scheduled events: six station-keeping maneuvers and one maneuver to avoid flying through the Earth's shadow (2007 November 11), followed by a small orbital correction 19 days later. The other interruption to observations occurred as a result of the failure of the primary transmitter used to telemeter data to Earth. WMAP was subsequently

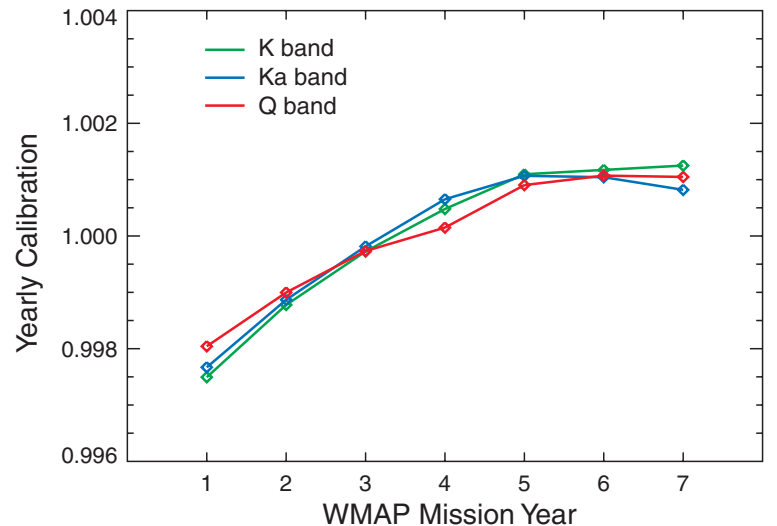

Figure 1. Measurements of the year-to-year calibration variation for $\mathrm{K}, \mathrm{Ka}$, and $\mathrm{Q}$ bands obtained by correlating the Galactic plane signal in the seven-year map to the signal in single-year sky maps. Note that the measured variations are consistent with the estimated absolute calibration uncertainty of $0.2 \%$. No significant variation is seen for the $\mathrm{V}$ - and $\mathrm{W}$-band maps.

reconfigured to use its backup transmitter and normal operations resumed with no performance degradation. On 2008 August 1 (day number 141) WMAP flew through the Moon's shadow, causing a $\approx 4 \%$ decrease in the incident solar flux lasting $\approx 6$ hr. WMAP remained in normal observing mode throughout this event, but data were excluded from sky map processing due to minor instrument thermal perturbations as described in Section 2.3.

\subsection{Calibration}

The algorithm used to calibrate the time-ordered data (TOD) is the same as was used for the five-year processing (Hinshaw et al. 2009). Calibration occurs in two steps. First, an hourly absolute gain and baseline are determined. The absolute calibration is based on the CMB monopole temperature (Mather et al. 1999) and the velocity-dependent dipole resulting from WMAP's orbit about the solar system barycenter. The calibration is performed iteratively, since removing the fixed sky signals arising from the barycentric $\mathrm{CMB}$ signal and foregrounds requires values of both the gain and baseline solutions. The only change made to this step of the calibration procedure is that the initial value for the barycentric $\mathrm{CMB}$ dipole signal has been updated to agree with the value determined from the five-year analysis.

The second step in the calibration procedure consists of fitting the hourly radiometer gain values to a model which relates the gain to measured values of radiometer parameters. The functional form of the gain model is the same as for the five-year data release, but the fitting procedure now utilizes all seven years of observational data. The seven-year mean changes in the calibration are presented in Table 1 . Note that the rms of the fractional calibrations change, $\Delta G / G$, is only $0.05 \%$. The absolute calibration accuracy is estimated to be $0.2 \%$, unchanged from the previously published value.

For the $\mathrm{K}, \mathrm{Ka}$, and $\mathrm{Q}$ bands, there is a small but significant year-to-year variation in the WMAP calibration that can be seen as variations of the Galactic plane brightness in yearly sky maps. This has been measured by correlating each yearly map against the seven-year map for pixels at $|b|<10^{\circ}$. A slope and offset is fit to each correlation and the slope values are adopted as yearly calibration variations. These are shown in Figure 1 and are consistent with the $0.2 \%$ absolute calibration uncertainty. 


\subsection{Gaps in Observations}

The WMAP instrument provides the highest quality data when the observatory is scanning the sky in its nominal observing mode and the instrument thermal environment is stable. Periodically, conditions arise that result in one or both of these conditions not being met. These situations arise from scheduled station-keeping maneuvers, and unscheduled events, such as solar flares or on-board equipment malfunctions. Data taken during these periods are excluded from the sky map processing to ensure the highest quality data products. Previously, potentially corrupted data segments were identified by manually inspecting the event logs and instrument thermal trend plots. The current data release uses a more objective automated procedure to identify the unusable data segments. The automated procedure was designed to approximate the manual procedure, but small differences will occur in the sky coverage between the first five years of the current data release and the previous fiveyear data release. Using the new procedure WMAP still achieves an overall observing efficiency of $\approx 98.4 \%$, down slightly from its previous value of $\approx 99 \%$.

The automated procedure identifies suspect events based on the time derivative of the focal plane assembly (FPA) temperature. The measured FPA temperature is averaged over one hour intervals, and the time derivative is formed by differencing successive values of these averages. Suspect thermal events are delineated by the times at which the magnitude of this derivative initially exceeds then finally falls below a threshold value. The threshold has been chosen to be five times the rms deviation of the temperature derivative signal occurring during normal observing periods, and corresponds to a value of $\approx 0.75 \mathrm{mK} \mathrm{hr}^{-1}$. In situations where the observatory was taken out of normal observing mode (e.g., during a scheduled station-keeping maneuver), the duration of the event is lengthened if needed to encompass the entire time the observatory was not in normal observing mode. For each event, data from $1.2 \mathrm{hr}$ before the event began to $7.25 \mathrm{hr}$ after the event ended are excluded from sky map processing.

\subsection{Planet Masking}

In the one-, three-, and five-year data analyses, observations when the boresight of each telescope beam fell within 1.5 of Mars, Jupiter, Saturn, Uranus, or Neptune were excluded from sky map processing, preventing contamination of the sky maps by emission from the planets. Subsequent analysis has shown that even with these exclusion criteria microwave emission from Jupiter could generate as much as a $75 \mu \mathrm{K}$ (in the $\mathrm{K}$ band) errant signal in a narrow annulus surrounding the region of excluded observations. Although there is no indication that this influenced the cosmological analysis, the radii used to exclude observations have been increased for the seven-year analysis, chosen to limit planetary leakage to less than $1 \mu \mathrm{K}$. However, given the uncertainty in the determination of the beam profiles at these levels, we adopt a conservative upper bound on possible planetary signal leakage into the TOD used for sky map production of $5 \mu \mathrm{K}$. The new values are displayed in Table 2.

\subsection{Expanded Diffuse Galactic Foreground Mask}

The mask for diffuse Galactic emission has been revised by including areas near the plane where the diffuse foreground cleaning algorithm appears to be less efficient than for the sky at large. These areas are found by performing a pixel-by-pixel $\chi^{2}$ test comparing null maps to cleaned $\mathrm{Q}-\mathrm{V}$ and $\mathrm{V}-\mathrm{W}$ maps with
Table 2

Data Exclusion Radii for the Planets

\begin{tabular}{lccccc}
\hline \hline Planet & \multicolumn{5}{c}{ Frequency Band } \\
\cline { 2 - 6 } & $\mathrm{K}$ & $\mathrm{Ka}$ & $\mathrm{Q}$ & $\mathrm{V}$ & $\mathrm{W}$ \\
\hline Mars & 2.0 & 1.5 & 1.5 & 1.5 & 1.5 \\
Jupiter & 3.0 & 2.5 & 2.5 & 2.2 & 2.0 \\
Saturn & 2.0 & 1.5 & 1.5 & 1.5 & 1.5 \\
Uranus & 2.0 & 1.5 & 1.5 & 1.5 & 1.5 \\
Neptune & 2.0 & 1.5 & 1.5 & 1.5 & 1.5 \\
\hline
\end{tabular}

resolution degraded from $\mathrm{r} 9^{15}$ to $\mathrm{r} 5$. All pixels with $\chi^{2}$ greater than four times that of the $\chi^{2}$ in the polar caps regions are cut. Small islands of cut pixels are eliminated from the cut if they contain 4 or fewer contiguous pixels. The two resulting masks based on $\mathrm{Q}-\mathrm{V}$ and $\mathrm{V}-\mathrm{W}$ analyses, respectively, are combined and promoted back to $\mathrm{r} 9$. The edges of the cut are smoothed by convolving the mask with a Gaussian of $3^{\circ}$ FWHM and cutting the result at a value of 0.5 . As the final step, the smoothed cut is combined with the five-year KQ85 or KQ75 cut (Gold et al. 2011). The resulting masks, termed KQ85y7 and KQ75y7, admit $78.3 \%$ and $70.6 \%$ of the sky, respectively, as compared to $81.7 \%$ and $71.6 \%$ for the five-year versions - a decrease of the admitted sky area by $3.4 \%$ of the full sky for KQ85 and $1.0 \%$ for KQ75.

\subsection{Map-making with Asymmetric Masking}

The most significant change in the current processing is the use of asymmetric masking in the iterative reconstruction of the sky maps. WMAP's differential design means that the TOD represents differences between the intensities of pairs of points on the sky observed by the two telescope beams. Reconstructing sky maps from differential data requires solving a set of linear equations that describe the relation between the differential TOD and the sky signal. Solution of this set of equations is performed iteratively, using sky maps from earlier iterations to alternately remove estimated sky signals for each beam from the TOD, leaving the value associated with the opposite beam which is then used to generate the next iteration of the sky map. This procedure becomes problematic when one of the beams is in a region of high intensity and the other is in a region of low intensity. Small errors arising from pixelization, residual pointing errors, beam ellipticity, or radiometer gain errors limit the degree to which the signal associated with the beam in the high emission region can be estimated. Such errors result in errant signals being introduced into sky map pixels associated with the beam in the low-intensity region. This potential problem is circumvented by masking portions of the data when such errors are likely to be introduced.

In the WMAP first-year results, an asymmetric masking procedure was used (Hinshaw et al. 2003). Asymmetric masking means that when one beam is in a high Galactic emission region (as determined by a processing mask; Limon et al. 2010) and the other beam is in a low Galactic emission region, only the pixel in the high emission region is iteratively updated. This allows for the reconstruction of full-sky maps while avoiding the situation that could produce an errant signal. The maps, $\mathbf{t}_{1}$, were obtained through a simple iterative solution of the linear

\footnotetext{
15 WMAP uses the HEALPix (Gorski et al. 2005) pixelization and labels resolution as $\mathrm{r} 4, \mathrm{r} 5, \mathrm{r} 9$, and $\mathrm{r} 10$ corresponding to HEALPix $N_{\text {side }}$ values of 16 , 32,512 , and 1024 , respectively.
} 
equation

$$
\mathbf{t}_{1}=\mathbf{W} \mathbf{d}_{1}
$$

where $\mathbf{d}_{1}$ is the pre-whitened TOD and $\mathbf{W}=\left(\mathbf{M}^{\mathrm{T}} \mathbf{M}\right)^{-1} \cdot \mathbf{M}^{\mathrm{T}}$. The matrix $\mathbf{M}$ is the mapping function, which has $N_{p}$ columns and $N_{t}$ rows, corresponding to the number of sky map pixels and TOD points, respectively. When the map processing includes polarization degrees of freedom $N_{p}$ is four times the number of map pixels, corresponding to the Stokes $I, Q$, and $U$ components and a spurious component, $S$, used to absorb effects arising from bandpass differences between the two radiometers comprising each differencing assembly (DA). See Jarosik et al. (2007) for a description of the implementation of the spurious mode, $S$. Multiplying a sky map vector by $\mathbf{M}$ effectively generates the TOD that would be obtained if WMAP observed a sky corresponding to the map vector. The sky maps obtained by solving Equation (1) are an unbiased representation of the true sky signal, but do not treat the noise terms optimally.

The WMAP three-year (Jarosik et al. 2007) and five-year (Hinshaw et al. 2009) analyses generated maximum likelihood map solutions using a conjugate gradient iterative technique. The maximum likelihood estimate of the sky map, $\tilde{\mathbf{t}}$, is

$$
\tilde{\mathbf{t}}=\left(\mathbf{M}^{\mathrm{T}} \mathbf{N}^{-1} \mathbf{M}\right)^{-1} \cdot\left(\mathbf{M}^{\mathrm{T}} \mathbf{N}^{-1} \mathbf{d}\right)
$$

where $\mathbf{d}$ is the calibrated but unfiltered TOD and $\mathbf{N}^{-1}$ is the inverse of the radiometer noise covariance matrix. Solution of this equation involves a direct evaluation of the last term of Equation (2) once, resulting in a map $\mathbf{t}_{\mathbf{0}}=\mathbf{M}^{\mathrm{T}} \mathbf{N}^{-1} \mathbf{d}$, followed by the iterative solution of the equation

$$
\tilde{\mathbf{t}}=\left(\mathbf{M}^{\mathrm{T}} \mathbf{N}^{-1} \mathbf{M}\right)^{-1} \cdot \mathbf{t}_{\mathbf{0}}
$$

Solving this equation with the conjugate gradient method requires the use of symmetric masking since this method can only be used when the matrix multiplying $\mathbf{t}_{\mathbf{0}}$ (in this case $\left.\left(\mathbf{M}^{\mathrm{T}} \mathbf{N}^{-1} \mathbf{M}\right)^{-1}\right)$ is symmetric. Symmetric masking means that if either beam is in a region of high Galactic emission, neither pixel is updated. Symmetric masking is implemented by simply replacing all occurrences of the full-sky mapping matrix, $\mathbf{M}$, with a masked version of the matrix in Equations (2) and (3). Since symmetric masking produces sky maps with no information in the high Galactic emission regions, in previous analyses two sets of sky maps were generated, symmetrically masked maps, and full-sky maps incorporating no masking. Data from full-sky maps were used to fill in regions of the symmetrically masked maps which contained no data. This procedure was a significant advancement over the iterative procedure used in the WMAP first-year results, in that it produced maximum likelihood maps and allowed for direct determination of the level of convergence of the solution. Its major disadvantages are that two sets of maps have to be generated then pieced together, and there is no simple method of calculating a pixel-pixel noise matrix which describes the noise covariance between pixels obtained from the two different input maps. The pixel-pixel noise covariance matrix delivered with the data release only applied to the pixels in the low-Galactic emission regions, and only diagonal weights are used to describe the noise properties in the regions of high Galactic emission.

Incorporating asymmetric masking into the maximum likelihood sky maps solution requires solving the equation

$$
\tilde{\mathbf{t}}=\left(\mathbf{M}_{\mathrm{am}}^{\mathbf{T}} \mathbf{N}^{-1} \mathbf{M}\right)^{-1} \cdot\left(\mathbf{M}_{\mathrm{am}}^{\mathbf{T}} \mathbf{N}^{-1} \mathbf{d}\right)
$$

where $\mathbf{M}_{\mathrm{am}}^{\mathrm{T}}$ is the mapping matrix incorporating asymmetric masking and $\mathbf{M}$ is the unmasked mapping matrix. Again, this equation is solved by direct evaluation of the last term,

$$
\mathbf{t}_{0}=\mathbf{M}_{\mathrm{am}}^{\mathrm{T}} \mathbf{N}^{-1} \mathbf{d}
$$

once, followed by an iterative solution of the equation

$$
\tilde{\mathbf{t}}=\left(\mathbf{M}_{\mathrm{am}}^{\mathrm{T}} \mathbf{N}^{-1} \mathbf{M}\right)^{-1} \cdot \mathbf{t}_{\mathbf{0}} .
$$

Note that the matrix $\mathbf{M}_{\mathrm{am}}^{\mathrm{T}} \mathbf{N}^{-1} \mathbf{M}$ is not symmetric, and therefore this equation cannot be solved using a simple conjugate gradient algorithm as was done previously. A bi-conjugate gradient stabilized method (Barrett et al. 1994) was chosen to solve this equation since it offered good convergence properties and was straightforward to implement, requiring relatively minor changes to the existing procedures. This technique was verified by reconstructing input maps to numerical precision from simulated noise-free TOD. Utilizing asymmetric masking eliminates both of the aforementioned shortcomings of the simple conjugate gradient method-each DA only requires a single map solution, and a pixel-pixel inverse noise covariance matrix can be generated which describes the noise correlation between all the pixels in the map.

\subsection{Calculation of the Pixel-Pixel Noise Covariance Matrix, $\Sigma$}

The pixel-pixel noise covariance matrix is given by $\boldsymbol{\Sigma}=$ $\left\langle\tilde{\mathbf{t}}_{\mathrm{n}} \tilde{\mathbf{t}}_{\mathrm{n}}^{\mathrm{T}}\right\rangle$, where $\tilde{\mathbf{t}}_{\mathrm{n}}$ is the noise component of a reconstructed sky map and the brackets denote an ensemble average. The TOD, $\mathbf{d}$, used to generate the map may be written as the sum of a signal term and a noise term

$$
\mathbf{d}=\mathbf{M t}+\mathbf{n}
$$

where $\mathbf{M}$ is the full-sky (unmasked) mapping matrix, $\mathbf{t}$ is a vector representing the input sky signal, and $\mathbf{n}$ is a vector corresponding to the radiometer noise, its covariance being described as

$$
\mathbf{N}=\left\langle\mathbf{n n}^{\mathrm{T}}\right\rangle .
$$

The noise component of the maximum likelihood asymmetrically masked map solution may be written as

$$
\tilde{\mathbf{t}}_{\mathrm{n}}=\left(\mathbf{M}_{\mathrm{am}}^{\mathbf{T}} \mathbf{N}^{-1} \mathbf{M}\right)^{-1} \cdot\left(\mathbf{M}_{\mathrm{am}}^{\mathbf{T}} \mathbf{N}^{-1} \mathbf{n}\right) .
$$

The noise covariance matrix becomes

$$
\begin{aligned}
\boldsymbol{\Sigma}= & \left\langle( \mathbf { M } _ { \mathrm { am } } ^ { \mathrm { T } } \mathbf { N } ^ { - 1 } \mathbf { M } ) ^ { - 1 } ( \mathbf { M } _ { \mathrm { am } } ^ { \mathrm { T } } \mathbf { N } ^ { - 1 } \mathbf { n } ) \cdot \left[\left(\mathbf{M}_{\mathrm{am}}^{\mathrm{T}} \mathbf{N}^{-1} \mathbf{M}\right)^{-1}\right.\right. \\
& \left.\left.\times\left(\mathbf{M}_{\mathrm{am}}^{\mathrm{T}} \mathbf{N}^{-1} \mathbf{n}\right)\right]^{\mathrm{T}}\right\rangle \\
= & \left\langle\left(\mathbf{M}_{\mathrm{a} m}^{\mathrm{T}} \mathbf{N}^{-1} \mathbf{M}\right)^{-1}\left(\mathbf{M}_{\mathrm{am}}^{\mathrm{T}} \mathbf{N}^{-1} \mathbf{n}\right) \cdot\left(\mathbf{n}^{\mathrm{T}} \mathbf{N}^{-1} \mathbf{M}_{\mathrm{am}}\right)\right. \\
& \left.\times\left(\mathbf{M}^{\mathrm{T}} \mathbf{N}^{-1} \mathbf{M}_{\mathrm{am}}\right)^{-1}\right\rangle \\
= & \left(\mathbf{M}_{\mathrm{am}}^{\mathrm{T}} \mathbf{N}^{-1} \mathbf{M}\right)^{-1}\left(\mathbf{M}_{\mathrm{am}}^{\mathrm{T}} \mathbf{N}^{-1}\right) \cdot\left(\left\langle\mathbf{n} \mathbf{n}^{\mathrm{T}}\right\rangle \mathbf{N}^{-1}\right) \mathbf{M}_{\mathrm{am}} \\
& \times\left(\mathbf{M}^{\mathrm{T}} \mathbf{N}^{-1} \mathbf{M}_{\mathrm{am}}\right)^{-1} \\
= & \left(\mathbf{M}_{\mathrm{am}}^{\mathrm{T}} \mathbf{N}^{-1} \mathbf{M}\right)^{-1} \cdot\left(\mathbf{M}_{\mathrm{am}}^{\mathrm{T}} \mathbf{N}^{-1} \mathbf{M}_{\mathrm{am}}\right) \cdot\left(\mathbf{M}^{\mathrm{T}} \mathbf{N}^{-1} \mathbf{M}_{\mathrm{am}}\right)^{-1} .
\end{aligned}
$$


Table 3

Seven-year Transmission Imbalance Coefficients

\begin{tabular}{lrcccc}
\hline \hline Radiometer & \multicolumn{1}{c}{$x_{\text {im }}$} & Uncertainty & Radiometer & $x_{\text {im }}$ & Uncertainty \\
\hline K11 & -0.00063 & 0.00022 & K12 & 0.00539 & 0.00010 \\
Ka11 & 0.00344 & 0.00017 & Ka12 & 0.00153 & 0.00011 \\
Q11 & 0.00009 & 0.00051 & Q12 & 0.00393 & 0.00025 \\
Q21 & 0.00731 & 0.00058 & Q22 & 0.01090 & 0.00116 \\
V11 & 0.00060 & 0.00025 & V12 & 0.00253 & 0.00068 \\
V21 & 0.00378 & 0.00033 & V22 & 0.00331 & 0.00106 \\
W11 & 0.00924 & 0.00207 & W12 & 0.00145 & 0.00046 \\
W21 & 0.00857 & 0.00227 & W22 & 0.01167 & 0.00154 \\
W31 & -0.00073 & 0.00062 & W32 & 0.00465 & 0.00054 \\
W41 & 0.02314 & 0.00461 & W42 & 0.02026 & 0.00246 \\
\hline
\end{tabular}

Notes. Transmission imbalance coefficients, $x_{\mathrm{im}}$, and their uncertainties determined from the seven-year observational data.

In practice, the terms $\mathbf{M}_{\mathrm{am}}^{\mathrm{T}} \mathbf{N}^{-1} \mathbf{M}$ and $\mathbf{M}_{\mathrm{am}}^{\mathrm{T}} \mathbf{N}^{-1} \mathbf{M}_{\mathrm{am}}$ are evaluated at $\mathrm{r} 4$ with the $\mathbf{N}^{-1}$ normalized to unity at zero lag, and the inverse pixel-pixel noise covariance matrix is generated by forming the product

$$
\boldsymbol{\Sigma}^{-1}=\left(\mathbf{M}^{\mathrm{T}} \mathbf{N}^{-1} \mathbf{M}_{\mathrm{am}}\right) \cdot\left(\mathbf{M}_{\mathrm{am}}^{\mathrm{T}} \mathbf{N}^{-1} \mathbf{M}_{\mathrm{am}}\right)^{-1} \cdot\left(\mathbf{M}_{\mathrm{am}}^{\mathrm{T}} \mathbf{N}^{-1} \mathbf{M}\right) .
$$

Regions of the $\Sigma^{-1}$ matrix corresponding to combinations of Stokes $I, Q$, and $U$ are then converted to noise units by dividing by the appropriate combinations of $\sigma_{0}(I)$ and $\sigma_{0}(Q, U)$ given in Table 1.

The term $\mathbf{M}_{\mathrm{am}}^{\mathrm{T}} \mathbf{N}^{-1} \mathbf{M}_{\mathrm{am}}$ is numerically inverted and is used as the source for the off-diagonal terms of the preconditioner for the bi-conjugate gradient stabilized algorithm.

\section{8. $N_{\mathrm{obs}}$ Fields of the Maps}

The asymmetric masking used to generate sky maps requires a new procedure for calculating the effective number of observations, $N_{\text {obs }}$, for each map pixel. In previous data releases, the effective number of observations was calculated by accumulating the number of TOD points falling within each pixel, weighted by the appropriate transmission imbalance coefficients, $x_{\mathrm{im}}$, and polarization projection factors $(\sin 2 \gamma, \cos 2 \gamma)$. These values corresponded to the diagonal elements of the inverse pixel-pixel noise covariance matrix $\boldsymbol{\Sigma}^{-1}=\mathbf{M}^{\mathrm{T}} \mathbf{N}^{-1} \mathbf{M}$ (Jarosik et al. 2007, Equation (26)), evaluated assuming white radiometer noise, i.e., $\mathbf{N}^{-1}=\mathbf{I}$. The simple procedure for evaluating these terms is not applicable to the form of $\boldsymbol{\Sigma}^{-1}$ for the asymmetrically masked maps, Equation (14). The $N_{\text {obs }}$ fields of the r9 and r10 sky maps were generated by evaluating the diagonal elements of Equation (14) with $\mathbf{N}^{-1}=\mathbf{I}$ using sparse matrix techniques. The $N_{\text {obs }}$ fields of the r4 sky maps are described in Section 2.10.

\subsection{Projecting Transmission Imbalance Modes from the $\boldsymbol{\Sigma}^{-1}$ Matrices}

As described in Jarosik et al. (2007), errors in the determination of the transmission imbalance parameters, $x_{\mathrm{im}}$, are a potential source of systematic artifacts in the reconstructed sky maps. These time-independent parameters, which specify the difference between the transmission between the A-side and B-side optical systems for each radiometer, are measured from the flight data, and are presented in Table 3. To prevent biasing the cosmological analyses, sky map modes that can be excited by these measurement errors are identified and projected from the $\Sigma^{-1}$ matrices.
The procedure follows the same method as used in the three- and five-year analyses and consists of generating a oneyear span of simulated TOD using the nominal values of the loss imbalance parameters. Sky maps are processed from this archive using the input values of $x_{\mathrm{im}}$ and altered values to simulate errors in the measured values of the coefficients. The differences between the resultant maps are used to identify map modes resulting from processing the data with altered $x_{\mathrm{im}}$ values. Two modifications have been made to this procedure relative to the previous analyses. (1) The simulated sky maps are formed at $\mathrm{r} 4$ by direct evaluation of Equations (5) and (6) at $\mathrm{r} 4$. This change was adopted to eliminate contamination of the transmission imbalance templates by the poorly measured sky map modes associated with monopoles in the Stokes $I$ and spurious mode $S$ sky maps. It was found that varying the $x_{\mathrm{im}}$ factors produced large changes in the amplitudes of these poorly measured modes in addition to exciting the mode associated with the loss imbalance. Through utilization of a singular value decomposition it was possible to null the poorly measured modes associated with the aforementioned monopoles while preserving the modes associated with the transmission imbalance while evaluating Equation (6). (2) Transmission imbalance modes were evaluated both for the case when the $x_{\mathrm{im}}$ for both radiometers comprising each DA were increased $20 \%$ above their measured values, and for the case when the $x_{\text {im }}$ value for radiometer 1 was increased by $10 \%$ and that of radiometer 2 decreased by $10 \%$. (Previously the only combination used was that in which both $x_{\mathrm{im}}$ values were increased.) For six of the DAs very similar sky map modes were generated by both sets of $x_{\mathrm{im}}$, while for the V2, W1, W2, and W4 somewhat different modes were generated for the two different sets. As a result, only one mode was projected from the $\mathrm{K} 1, \mathrm{Ka} 1, \mathrm{Q} 1, \mathrm{Q} 2$, and $\mathrm{W} 3$ matrices, while two modes were projected out of the V2, W1, W2, and W4 matrices. In each case, the modes were removed from $\Sigma^{-1}$ following the method described in Jarosik et al. (2007).

\subsection{Low-resolution Single-year Map Generation}

The low-resolution sky maps were generated by performing an inverse noise weighted degradation of the high-resolution maps that takes into account the intra-pixel noise correlations of the high-resolution input maps. The weight matrix for the polarization (Stokes $Q$ and $U$ ) and spurious mode $(S)$ of each high-resolution map pixel, $\mathrm{p} 9$, is given by

$$
\mathbf{N}_{\mathrm{obs}}(\mathrm{p} 9)=\left(\begin{array}{lll}
N_{\mathrm{QQ}} & N_{\mathrm{QU}} & N_{\mathrm{QS}} \\
N_{\mathrm{QU}} & N_{\mathrm{UU}} & N_{\mathrm{SU}} \\
N_{\mathrm{QS}} & N_{\mathrm{SU}} & N_{\mathrm{SS}}
\end{array}\right)
$$

where each element $N_{\mathrm{XY}}$ is an element of $\boldsymbol{\Sigma}^{-1}$ (Equation (14)) evaluated as described in Section 2.8. For each DA year combination, the $Q, U, S$ map sets were generated as

$$
\begin{gathered}
\left(\begin{array}{c}
Q_{\mathrm{p} 4} \\
U_{\mathrm{p} 4} \\
S_{\mathrm{p} 4}
\end{array}\right)=\left(\mathbf{N}_{\mathrm{obs}}^{\mathrm{tot}}(\mathrm{p} 4)\right)^{-1} \sum_{\mathrm{p} 9 \in \mathrm{p} 4} \mathbf{N}_{\mathrm{obs}}(\mathrm{p} 9)\left(\begin{array}{c}
Q_{\mathrm{p} 9} \\
U_{\mathrm{p} 9} \\
S_{\mathrm{p} 9}
\end{array}\right), \\
\mathbf{N}_{\mathrm{obs}}^{\mathrm{tot}}(\mathrm{p} 4)=\sum_{\mathrm{p} 9 \in p 4} \mathbf{N}_{\mathrm{obs}}(\mathrm{p} 9) .
\end{gathered}
$$

The $N_{\text {obs }}$ fields of the low-resolution sky maps contain the diagonal elements of the corresponding portions of the $\boldsymbol{\Sigma}^{-1}$ matrices as described in Section 2.7 before the scaling by $\sigma_{0}$ is applied. 


\subsection{Low-resolution Multi-year Map Generation}

The single-year single-DA maps were combined to form a seven-year map for each DA and seven-year maps for each frequency band. The individual low-resolution maps were inverse noise weighted using the $\tilde{\boldsymbol{\Sigma}}$ matrices (Section 2.9), scaled to reflect the noise level of each DA. The weighted polarization maps were formed as

$$
\begin{gathered}
\left(\begin{array}{c}
Q \\
U
\end{array}\right)=\tilde{\Sigma}^{\mathrm{tot}} \sum_{\mathrm{yr}, \mathrm{DA}} \tilde{\boldsymbol{\Sigma}}_{\mathrm{yr}, \mathrm{DA}}^{-1}\left(\begin{array}{c}
Q \\
U
\end{array}\right)_{\mathrm{yr}, \mathrm{DA}}, \\
\tilde{\boldsymbol{\Sigma}}^{\mathrm{tot}}=\left(\sum_{\mathrm{yr}, \mathrm{DA}} \tilde{\boldsymbol{\Sigma}}_{\mathrm{yr}, \mathrm{DA}}^{-1}\right)^{-}
\end{gathered}
$$

where ()$^{-}$represents a pseudo-inverse of the sum in parenthesis. This summation is performed over the year/DA combinations to be included in the final map. For $\mathrm{K}$ and $\mathrm{Ka}$ bands, the pseudo-inverse is calculated by performing a singular value decomposition of the sum of the $Q U \times Q U \tilde{\boldsymbol{\Sigma}}^{-1}$ matrices and inverting all its eigenvalues except for the smallest, which is set to zero. This procedure de-weights the one nearly singular mode associated with the monopoles in the $I$ and $S$ maps. No modes are removed from the Q-, V-, and W-band maps.

\section{BEAM MAPS AND WINDOW FUNCTIONS}

The seven-year WMAP beams and window functions result from a refinement of the data reduction methods used for the five-year beams, with no major changes in the processing steps.

Briefly, in the five-year analysis, the beam maps were accumulated from TOD samples with Jupiter in either the A or the B side beam. Sky background was subtracted using the sevenyear full-sky maps, with the band-dependent Jupiter exclusion radii (Section 2.4). Because of the motion of Jupiter, and the numerous Jupiter observing seasons, ${ }^{16}$ the sky coverage of the maps was $100 \%$ in spite of this masking.

As far as possible, the beam transfer functions $b_{\ell}$ in Fourier space were computed directly from Jupiter data. However, at low signal levels, some information was incorporated from beam models. The A- and B-side Jupiter data were fitted separately by a physical optics model comprising feed horns with fixed profiles, together with primary and secondary mirrors described by fit parameters. The geometrical configuration of these components was fixed. The fitted parameters described small distortions of the mirror surface shapes.

The beam models were combined with the Jupiter data at low signal levels by a hybridization algorithm. This process was optimized to yield the minimum uncertainty in beam solid angle, given the instrumental noise, under the conservative assumption that the systematic error in the beam model was $100 \%$. Details, as well as a summary of beam processing in earlier data releases, were given by Hill et al. (2009).

In addition to the use of three more seasons of Jupiter data, the following refinements, described below in more detail, have been made for the seven-year beam processing.

1. Subtraction of background flux from Jupiter observations is improved by expanding the exclusion radius for sky maps (Section 2.4).

16 A "season" is a $\approx 45$ day period when a planet falls within the scan pattern of WMAP. Each planet typically has two seasons per year.
2. The beam modeling procedure was modified by adding additional terms to the description of the secondary mirror figure to approximate a hypothetical tip/tilt.

3. The rate of convergence of the physical optics model is improved by correcting an orthogonalization error in the conjugate gradient code.

4. The physical optics fit is driven harder in two senses.

(a) A smaller change in $\chi^{2}$ between conjugate gradient descent steps is required to declare convergence.

(b) The model is fitted over a wider field of view in the observed beam map.

The change in beam profiles and transfer functions between the five- and seven-year analyses is conveniently summarized by a comparison of solid angles for the 10 DAs, given in Table 4. The solid angle increases of $0.8 \%$ in $\mathrm{K} 1$ and $0.4 \%$ in $\mathrm{Ka} 1$ are the result of the improved background estimates for the Jupiter observations. The solid angle changes for the Q1-W4 DAs have multiple causes. First, the instrumental noise in the Jupiter samples is different in detail. Second, the beam models have been refitted and differ slightly from the five-year versions. Third, the increased signal-to-noise ratio of the Jupiter data in the beam wings means that for some DAs, the hybrid threshold is optimized to a lower gain with seven years of data. This value is the limit below which observed intensity values in the Jupiter TOD are replaced with computed values from the beam model. For the five-year data analysis, the hybrid thresholds were 3, 4, 6,8 , and $11 \mathrm{dBi}$, respectively, for bands $\mathrm{K}, \mathrm{Ka}, \mathrm{Q}, \mathrm{V}$, and $\mathrm{W}$ (Hill et al. 2009). For the seven-year data analysis, the $\mathrm{V}$ and $\mathrm{W}$ thresholds are 7 and $10 \mathrm{dBi}$, respectively, while those for $\mathrm{K}, \mathrm{Ka}$, and $Q$ are unchanged.

As shown in the table, the aggregate solid angle changes for $\mathrm{V}$ and $\mathrm{W}$, which are the bands used in the high- $\ell \mathrm{TT}$ power spectrum, are $\sim 0.1 \%$ or less. Table 4 also gives the current values of the forward gain $G_{m}$ and of the factor $\Gamma_{\mathrm{ff}}$ for converting antenna temperature to flux in Jy. New hybrid beam profiles and beam transfer functions $b_{\ell}$ are available online from the Legacy Archive for Microwave Background Data Analysis (LAMBDA).

\subsection{Seven-year Beam Model Fitting}

\subsubsection{Secondary Tip/Tilt}

One change in the seven-year beam computations is to introduce approximate tip/tilt terms for the secondary mirror into the physical optics model. This change is motivated by the stable $\sim 0.1$ offset in the collective B-side boresight pointings, as compared to pre-flight expectations (Hill et al. 2009). In previous beam fits, this offset was absorbed by two free-floating nuisance parameters.

The introduction of secondary tip/tilt is a heuristic attempt to improve the fidelity of the beam model. Because a displacement in beam pointings could result from various small mechanical displacements in the instrument, or from a combination of them, the beams contain too little information to support a mechanical analysis. We emphasize that the boresight pointings on both the $A$ and B sides are equally stable since launch, to $<10^{\prime \prime}$ (Jarosik et al. 2007), an estimate that is confirmed by the full seven-year Jupiter data.

For convenience, we approximate tip/tilt as a purely planar surface distortion of the secondary mirror, in addition to the Bessel modes used previously. The pivot line is allowed to vary by including a term for scalar displacement together with tip/tilt slopes in two orthogonal directions. 
Table 4

WMAP Seven-year Main Beam Parameters

\begin{tabular}{|c|c|c|c|c|c|c|c|}
\hline $\mathrm{DA}$ & $\begin{array}{r}\Omega_{7 \mathrm{yr}}^{S \text { a }} \\
(\mathrm{sr})\end{array}$ & $\begin{array}{c}\Delta\left(\Omega_{7 \mathrm{yr}}^{S}\right) / \Omega^{S \mathrm{~b}} \\
(\%)\end{array}$ & $\begin{array}{c}\frac{\Omega_{7 \mathrm{yr}}^{S}}{\Omega_{5 \mathrm{yr}}^{S}}-1^{\mathrm{c}} \\
(\%)\end{array}$ & $\begin{array}{l}G_{m}^{\mathrm{d}} \\
(\mathrm{dBi})\end{array}$ & $\begin{array}{c}v_{\mathrm{eff}}^{\mathrm{ff}} \\
(\mathrm{GHz})\end{array}$ & $\begin{array}{l}\Omega_{\mathrm{eff}}^{\mathrm{ff}} \\
(\mathrm{sr})\end{array}$ & $\begin{array}{c}\Gamma_{\mathrm{ff}^{\mathrm{e}}}^{\mathrm{e}} \\
\left(\mu \mathrm{K} \mathrm{Jy}^{-1}\right)\end{array}$ \\
\hline \multicolumn{8}{|c|}{ For 10 Maps } \\
\hline K1 & $2.466 \times 10^{-4}$ & 0.6 & 0.8 & 47.07 & 22.72 & $2.519 \times 10^{-4}$ & 250.3 \\
\hline $\mathrm{Ka} 1$ & $1.442 \times 10^{-4}$ & 0.5 & 0.4 & 49.40 & 32.98 & $1.464 \times 10^{-4}$ & 204.5 \\
\hline Q1 & $8.832 \times 10^{-5}$ & 0.6 & -0.1 & 51.53 & 40.77 & $8.952 \times 10^{-5}$ & 218.8 \\
\hline $\mathrm{Q} 2$ & $9.123 \times 10^{-5}$ & 0.5 & -0.2 & 51.39 & 40.56 & $9.244 \times 10^{-5}$ & 214.1 \\
\hline V1 & $4.170 \times 10^{-5}$ & 0.4 & 0.0 & 54.79 & 60.12 & $4.232 \times 10^{-5}$ & 212.8 \\
\hline $\mathrm{V} 2$ & $4.234 \times 10^{-5}$ & 0.4 & -0.1 & 54.72 & 61.00 & $4.281 \times 10^{-5}$ & 204.4 \\
\hline W1 & $2.042 \times 10^{-5}$ & 0.4 & 0.2 & 57.89 & 92.87 & $2.044 \times 10^{-5}$ & 184.6 \\
\hline W2 & $2.200 \times 10^{-5}$ & 0.5 & -0.3 & 57.57 & 93.43 & $2.200 \times 10^{-5}$ & 169.5 \\
\hline W3 & $2.139 \times 10^{-5}$ & 0.5 & -0.5 & 57.69 & 92.44 & $2.139 \times 10^{-5}$ & 179.0 \\
\hline W4 & $2.007 \times 10^{-5}$ & 0.5 & 0.5 & 57.97 & 93.22 & $2.010 \times 10^{-5}$ & 186.4 \\
\hline \multicolumn{8}{|c|}{ For 5 Maps } \\
\hline$K$ & $2.466 \times 10^{-4}$ & 0.6 & 0.8 & 47.07 & 22.72 & $2.519 \times 10^{-4}$ & 250.3 \\
\hline$K a$ & $1.442 \times 10^{-4}$ & 0.5 & 0.4 & 49.40 & 32.98 & $1.464 \times 10^{-4}$ & 204.5 \\
\hline$Q$ & $8.978 \times 10^{-5}$ & 0.6 & -0.2 & 51.46 & 40.66 & $9.098 \times 10^{-5}$ & 216.4 \\
\hline$V$ & $4.202 \times 10^{-5}$ & 0.4 & -0.1 & 54.76 & 60.56 & $4.256 \times 10^{-5}$ & 208.6 \\
\hline$W$ & $2.097 \times 10^{-5}$ & 0.5 & 0.0 & 57.78 & 92.99 & $2.098 \times 10^{-5}$ & 179.3 \\
\hline
\end{tabular}

Notes.

a Solid angle in azimuthally symmetrized beam.

b Relative error in $\Omega^{S}$.

${ }^{c}$ Relative change in $\Omega^{S}$ between five-year and seven-year analyses.

${ }^{\mathrm{d}}$ Forward gain $=$ maximum of gain relative to isotropic, defined as $4 \pi / \Omega^{S}$. Values of $G_{m}$ in Table 2 of Hill et al. (2009) were taken from the physical optics model, rather than computed from the solid angle, and therefore do not obey this relation.

e Conversion factor to obtain flux density from the peak WMAP antenna temperature, for a free-free spectrum with $\beta=-2.1$.

Uncertainties in these factors are estimated as $0.6 \%, 0.5 \%, 0.6 \%, 0.5 \%$, and $0.7 \%$ for K-, Ka-, Q-, V-, and W-band DAs, respectively.

This parameter subspace has been explored using Monte Carlo beam simulations, which reveal a strong degeneracy between the scalar offset and a tilt in the spacecraft $Y Z$ plane, because both displacements move the illumination spot on the primary mirror in the up or down direction. Therefore, we have investigated a family of solutions by adopting an Ansatz for the mechanical constraints, such that (1) one edge of the secondary is allowed to pivot while pinned at the designed position, (2) the opposite edge undergoes a small displacement, and (3) the secondary mirror as a whole is rigid.

These constraints result in two discrete solutions for each secondary mirror; for each side of the instrument, the solution requiring the smaller mechanical displacement is chosen. The resulting edge displacement is $-4.4 \mathrm{~mm}$ on the B side, which corresponds to a hypothetical motion of one edge of the secondary mirror away from the secondary backing structure and toward the primary. Similarly, this method results in a hypothetical displacement of one edge of the A-side secondary by $0.2 \mathrm{~mm}$ away from the primary. However, as already explained, this mechanical interpretation is uncertain. For subsequent stages of beam modeling, the distortion coefficients mimicking tip/tilt and displacement of the secondary mirrors are held fixed.

\subsubsection{Fitting Process}

The overall fitting of beams progresses much as it did for the five-year data. On each of the A and B sides, the primary mirror is modeled with Fourier modes added to the nominal mirror figure. Similarly, the secondary mirror is modeled with Bessel modes. A complete fit is done using modes with spatial frequency on the mirror surface up to some maximum value. When convergence is achieved, the current best-fit parameters become the starting point for a new fit with a higher spatial frequency limit. The primary mirror spatial frequencies are indexed using an integer $k$, such that the wavelength of the mirror surface distortion is $280 \mathrm{~cm} / k$. The secondary modes are specified by the Bessel mode indexes $n$ and $k$. Details are given in Hill et al. (2009, Section 2.2.2). The maximum spatial frequencies fitted are defined by $k_{\max }=24$ on the primary and $n_{\max }=2$ on the secondary.

Because the Fourier modes are non-orthogonal over the circular domain of the primary mirror distortions, the fitting is actually done in an orthogonalized space (Hill et al. 2009). A bug in the orthogonalization code was corrected before the sevenyear analysis began; this bug only affected speed of convergence, not the definition of $\chi^{2}$, so the five-year results remain valid. However, this change made it practical to refit the beam models $\mathrm{ab}$ initio from the nominal mirror figures, i.e., with zero for all Fourier and Bessel coefficients, rather than beginning from the five-year solution.

The $\chi^{2}$ changes from the five-year to the seven-year beam models are shown in Table 5. The $\chi^{2}$ values shown for the fiveyear models are recomputed using seven-year data. It is clear from Table 5 that the overall improvement in the model for the 10 DAs collectively is driven primarily by the $\mathrm{W}$ band on the $\mathrm{A}$ side and the $\mathrm{V}$ and $\mathrm{W}$ bands on the $\mathrm{B}$ side.

Figure 2 shows model beam profiles from an example DA, W1, from both the five-year and the seven-year analyses. The A-side model has a similar profile in the five-year and seven-year fits, with small tradeoffs in fit quality at various radii. However, the B-side model shows a new feature in the seven-year fitting, namely, an elevated response at large radii and very low signal levels in the $\mathrm{V}$ and $\mathrm{W}$ bands. This feature is seen at radii larger than $2^{\circ}$ in the right-hand panel of Figure 2, where the seven-year profile (red) is very slightly higher than the five-year profile 
Table 5

Changes in $\chi^{2}$ from Five-year to Seven-year Beam Fits

\begin{tabular}{|c|c|c|c|}
\hline DA & $\begin{array}{c}\chi_{v}^{2} \\
\text { (Five-year) }\end{array}$ & $\begin{array}{c}\chi_{v}^{2} \\
\text { (Seven-year) }\end{array}$ & $\Delta \chi_{v}^{2}$ \\
\hline \multicolumn{4}{|c|}{ Side A } \\
\hline All & 1.060 & 1.049 & -0.011 \\
\hline K1 & 1.009 & 1.006 & -0.004 \\
\hline Ka1 & 1.012 & 1.016 & 0.004 \\
\hline Q1 & 1.078 & 1.074 & -0.003 \\
\hline Q2 & 1.094 & 1.102 & 0.008 \\
\hline V1 & 1.144 & 1.155 & 0.011 \\
\hline V2 & 1.162 & 1.171 & 0.008 \\
\hline W1 & 1.225 & 1.171 & -0.053 \\
\hline W2 & 1.239 & 1.151 & -0.088 \\
\hline W3 & 1.244 & 1.181 & -0.063 \\
\hline W4 & 1.208 & 1.156 & -0.052 \\
\hline \multicolumn{4}{|c|}{ Side B } \\
\hline All & 1.065 & 1.047 & -0.018 \\
\hline K1 & 1.022 & 1.017 & -0.005 \\
\hline Ka1 & 1.010 & 1.009 & -0.000 \\
\hline Q1 & 1.093 & 1.046 & -0.046 \\
\hline Q2 & 1.045 & 1.052 & 0.007 \\
\hline V1 & 1.288 & 1.171 & -0.117 \\
\hline V2 & 1.178 & 1.159 & -0.019 \\
\hline W1 & 1.226 & 1.197 & -0.029 \\
\hline W2 & 1.169 & 1.155 & -0.013 \\
\hline W3 & 1.223 & 1.203 & -0.021 \\
\hline W4 & 1.226 & 1.165 & -0.061 \\
\hline
\end{tabular}

(blue). The five-year and seven-year profiles for the example DA differ by a factor of $\sim 2-10$ in this region, although both are $\gtrsim 50 \mathrm{~dB}$ down from the beam peak.

Several attempts have been made using jumps in the fitting parameters (as in simulated annealing) to find a region of parameter space in the A-side fit that would lead to the existence of elevated tails similar to those on the B side. So far, such attempts have failed. The best A-side fit lacks the elevated-tail feature, while the best B-side fit has it.

Just as in the five-year analysis, none of the beam models meets a formal criterion for a good fit, since the number of degrees of freedom is of order $10^{5}$, or $10^{4}$ per DA, while the overall $\chi_{v}^{2}$ is $\sim 1.05$. However, the model beams are used only in a restricted role, more than $\sim 45 \mathrm{~dB}$ below the beam peak, so that the primary contribution to the beams and $b_{\ell}$ is directly from Jupiter data. As a result, the effect of any particular feature of the models is either omitted or diluted in the final result. However, the B-side model tail mentioned above increases the beam uncertainty slightly at large radii as compared to the fiveyear analysis.

The process for incorporating information from the models into the Jupiter-based beams is explained by Hill et al. (2009). This process is termed hybridization. Briefly, the beam profiles are integrated from Jupiter TOD. The two-dimensional coordinates of each TOD point within the beam model are determined. If the model gain is below a certain threshold, then the model gain is substituted for the Jupiter measurement. The thresholds are optimized for each DA by minimizing the uncertainty in the solid angle, under the assumption that the beam model is subject to a scaling uncertainty of $100 \%$. Final hybrid profiles combining the A and B sides are shown for the W1 DA, for both the five-year and seven-year analyses, in Figure 3.

The beam transfer function, $b_{\ell}$, is integrated directly from the hybrid beam profiles, and the error envelope for $b_{\ell}$ is computed

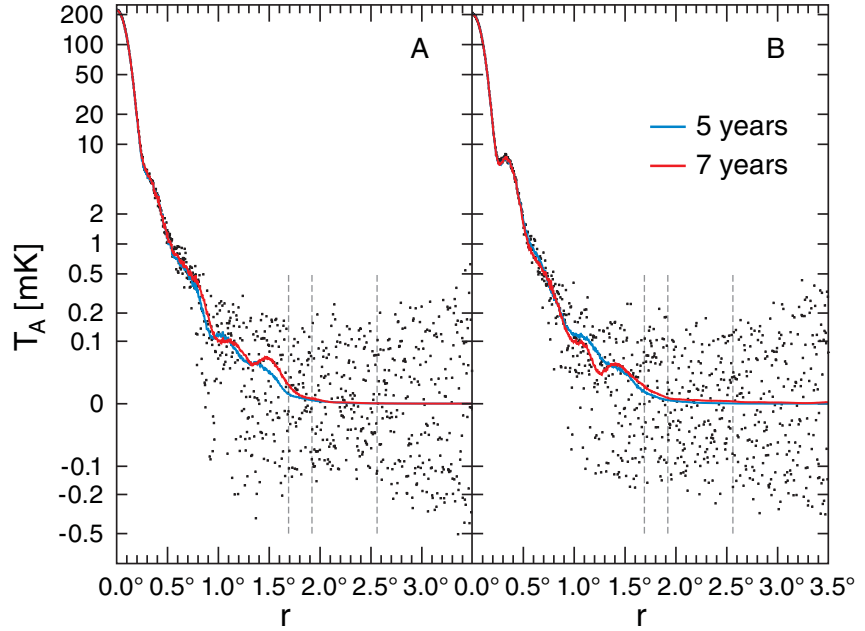

Figure 2. Physical optics beam models for the W1 DA on the A side (left) and B side (right) of the WMAP instrument. The ordinate is scaled by a sinh ${ }^{-1}$ function that provides a smooth transition between linear and logarithmic regimes. Blue: five-year models; red: seven-year models. Points: seven-year Jupiter beam data averaged in radial bins of $\Delta r=0.5$. Dashed lines: radii at which hybrid beam profiles consist of $90 \%, 50 \%$, and $10 \%$ Jupiter data, respectively, from smaller to larger radii. Model differences inside $r \sim 1.7$ are mostly suppressed in the hybrid beam profiles, whereas model differences outside $r \sim 2.6$ are mostly retained.

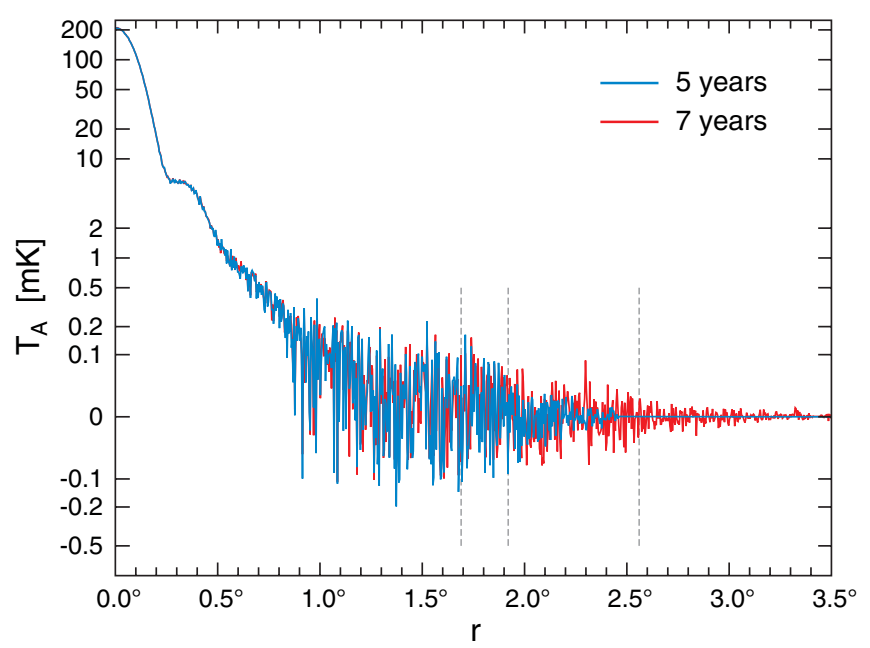

Figure 3. W1 hybrid beam profiles from five-year (blue) and seven-year (red) analysis, combined for the A and B sides. The ordinate is scaled by a sinh ${ }^{-1}$ function that provides a smooth transition between linear and logarithmic regimes. Dashed lines: radii at which hybrid beam profiles consist of $90 \%$, $50 \%$, and $10 \%$ Jupiter data, respectively, from smaller to larger radii. The noise shows that the use of Jupiter data extends effectively to larger radii in the seven-year analysis.

using a Monte Carlo method. These procedures are the same as in the five-year analysis (Hill et al. 2009). A comparison of five-year and seven-year $b_{\ell}$ and the corresponding uncertainties is shown in Figure 4.

\subsection{Flux Conversion Factors and Beam Solid Angles for Point Sources}

The conversion factor from peak antenna temperature to flux density for a point source is given by (Page et al. 2003a)

$$
\Gamma=c 2 / 2 k_{B} \Omega_{\text {eff }}\left(v_{\text {eff }}\right)^{2},
$$

where $\Omega_{\text {eff }}$ is the effective beam solid angle of the A and B sides combined and $v_{\text {eff }}$ is the effective band center frequency, both 

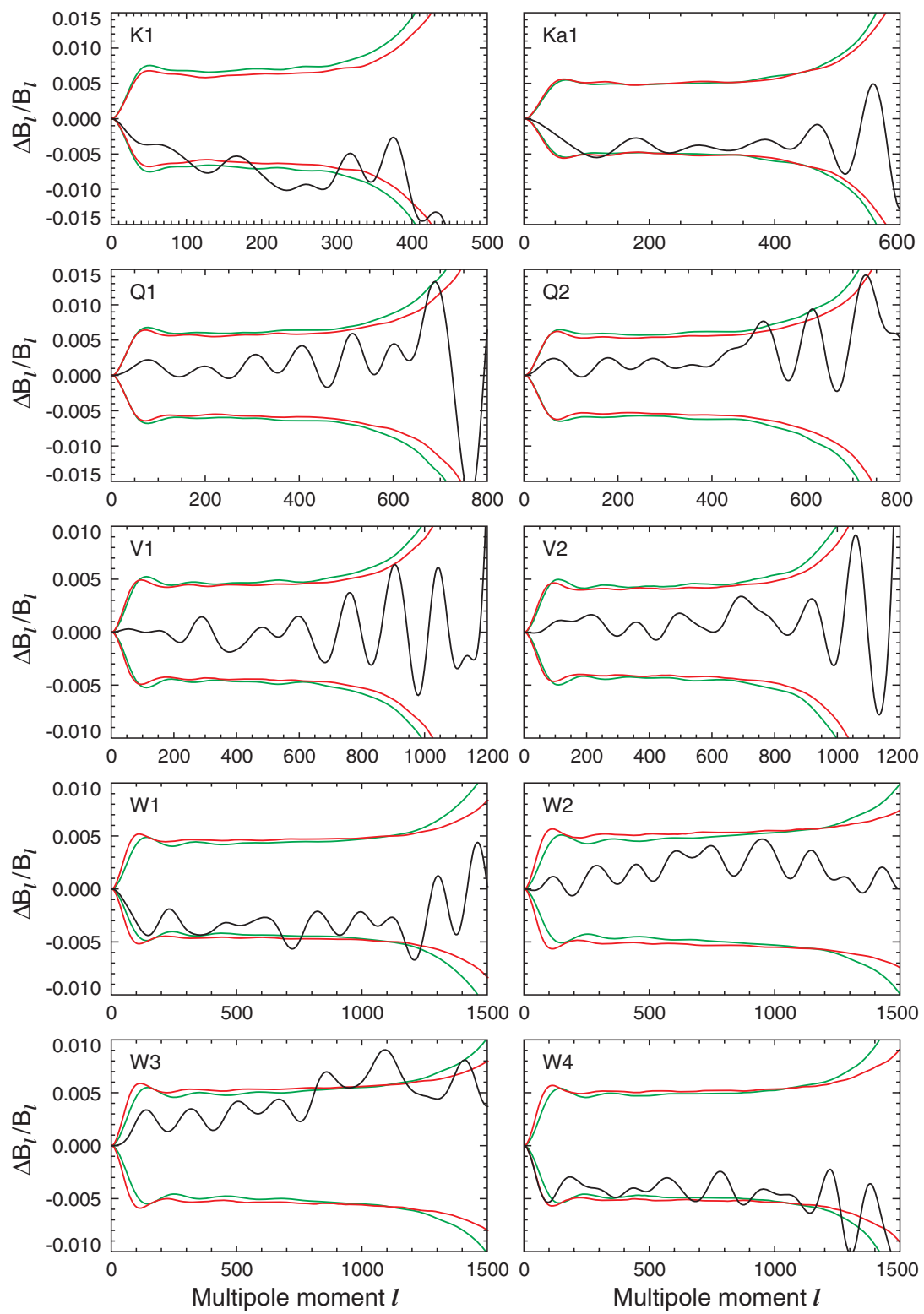

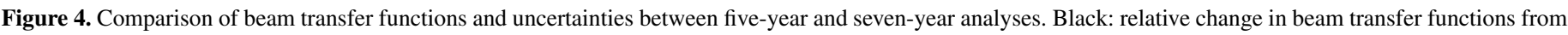

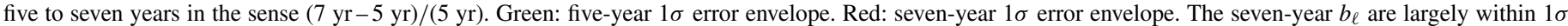

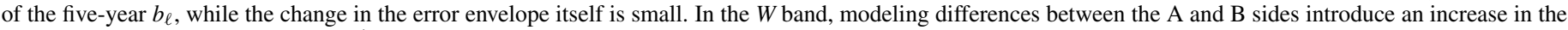
uncertainty plateau for multipoles $\ell \lesssim 1000$, whereas the small angle (high $\ell$ ) uncertainty is decreased for all bands.

of which depend on the source spectrum. New values of these quantities have been calculated that supersede previous results given for point sources.

For a point source with antenna temperature spectrum $T_{A} \propto$ $v^{\beta}$, the effective frequency is determined from

$$
v_{\mathrm{eff}}^{\beta}=\int f(v) G_{m}(v) v^{\beta} d v / \int f(v) G_{m}(v) d v,
$$

where $f(v)$ is the passband response and $G_{m}(v)$ is the forward gain. This is consistent with the definition used by Jarosik et al. (2003) for a beam-filling source, except in that case the forward gain is not included. Values of $v_{\text {eff }}(\beta)$ are calculated using pre-flight bandpass measurements and pre-flight GEMAC ${ }^{17}$ measurements of forward gain. A correction for scattering is

\footnotetext{
17 Goddard Electromagnetic Anechoic Chamber
}

applied to the GEMAC measurements,

$$
G_{m}(\nu)=G_{m}^{\operatorname{GEMAC}}(\nu) e^{\left(-(4 \pi \sigma / \lambda)^{2}\right)},
$$

where $\sigma$ is an effective rms primary mirror deformation whose value is set separately for each DA based on the degree of scattering estimated from the pass 4 physical optics modeling.

The effective solid angle is calculated as

$$
\Omega_{\mathrm{eff}}=\Omega(\text { Jupiter }) \Omega_{\mathrm{GEMAC}}(\beta) / \Omega_{\mathrm{GEMAC}}(\text { Jupiter}),
$$

where $\Omega$ (Jupiter) is the pass 4 beam solid angle determined from Jupiter observations and $\Omega$ (GEMAC) is the beam solid angle calculated for a given source spectrum using the scatteringcorrected GEMAC measurements. From Equation (25) of Page et al. (2003b),

$$
\Omega(\mathrm{GEMAC})=4 \pi \int f(v) v^{\beta} d v / \int f(v) G_{m}(\nu) v^{\beta} d \nu .
$$


Table 6

WMAP Seven-year CMB Dipole Parameters

\begin{tabular}{cccccc}
\hline \hline $\begin{array}{c}d_{x}{ }^{\mathrm{a}} \\
(\mathrm{mK})\end{array}$ & $\begin{array}{c}d_{y} \\
(\mathrm{mK})\end{array}$ & $\begin{array}{c}d_{z} \\
(\mathrm{mK})\end{array}$ & $\begin{array}{c}d^{\mathrm{b}} \\
(\mathrm{mK})\end{array}$ & $\begin{array}{c}l \\
\left({ }^{\circ}\right)\end{array}$ & $\begin{array}{c}b \\
\left({ }^{\circ}\right)\end{array}$ \\
\hline$-0.233 \pm 0.005$ & $-2.222 \pm 0.004$ & $2.504 \pm 0.003$ & $3.355 \pm 0.008$ & $263.99 \pm 0.14$ & $48.26 \pm 0.03$ \\
\hline
\end{tabular}

Notes. The measured values of the CMB dipole signal. These values are unchanged from the five-year values and are reproduced here for completeness.

a Cartesian components are give in Galactic coordinates. The listed uncertainties include the effects of noise, masking and residual foreground contamination. The $0.2 \%$ absolute calibration uncertainty should be added to these values in quadrature.

b The spherical components of the CMB dipole are given in Galactic coordinates and already include all uncertainty estimates, including the $0.2 \%$ absolute calibration uncertainty.

Values of $\nu_{\text {eff }}, \Omega_{\text {eff }}$, and $\Gamma$ for a point source with $\beta=-2.1$, typical for the sources in the WMAP point source catalog, are given in Table 2.

\section{SKY MAP DATA AND ANALYSIS}

The seven-year sky maps are consistent with the five-year maps apart from small effects related to the new processing methods. Figure 5 displays the seven-year band average Stokes I maps and the differences between these maps and the published five-year maps. The difference maps have been adjusted to compensate for the slightly different gain calibrations and dipole signals used in the different analysis. The small Galactic plane features in the K-, Ka-, and Q-band difference maps arise from the slightly different calibrations and small changes in the effective beam shapes. Pixels in the Galactic plane region are observed over a slightly smaller range of azimuthal beam orientation in the current data processing relative to previous analyses, resulting in slightly less azimuthal averaging and hence a slightly altered effective beam shape. The calibration gain changes relative to the five-year data release are small, all below $0.15 \%$ as indicated in Table 1 .

\subsection{The Temperature and Polarization Power Spectra}

\subsubsection{The Temperature Dipole and Quadrupole}

The five-year CMB dipole value was obtained using a Gibbs sampling method (Hinshaw et al. 2009) to estimate the dipole signal in both the five-year ILC map and foreground reduced maps. The uncertainties on the measured parameters were set to encompass both results as an estimate of the effect of residual foreground signals. The dipole measured from the seven-year data shows no significant changes from that obtained from the five-year data, so the best-fit dipole parameters remain unchanged from the five-year values, and are presented in Table 6.

The maximum likelihood value of magnitude of the CMB quadrupole is $l(l+1) C_{l} / 2 \pi=197_{-155}^{+2972} \mu \mathrm{K}^{2}(95 \%$ CL) (Larson et al. 2011) based on the analysis of the ILC map using the KQ85 mask. This value is essentially unchanged from the five-year results and lies below the most likely value predicted by the best-fit $\Lambda \mathrm{CDM}$ model. This value, however, is not particularly unlikely given the distribution of values predicted by the model, as described in Bennett et al. (2011).

Figure 6 displays the seven-year band average maps for Stokes $Q$ and $U$ components for all five WMAP frequency bands. Polarized Galactic emission is evident in all frequency bands. The smooth large angular scale features visible in the W-band maps, and to a lesser degree in the V-band maps, are the result of a pair of modes that are poorly constrained by the map-making procedure. While these dominate the appearance
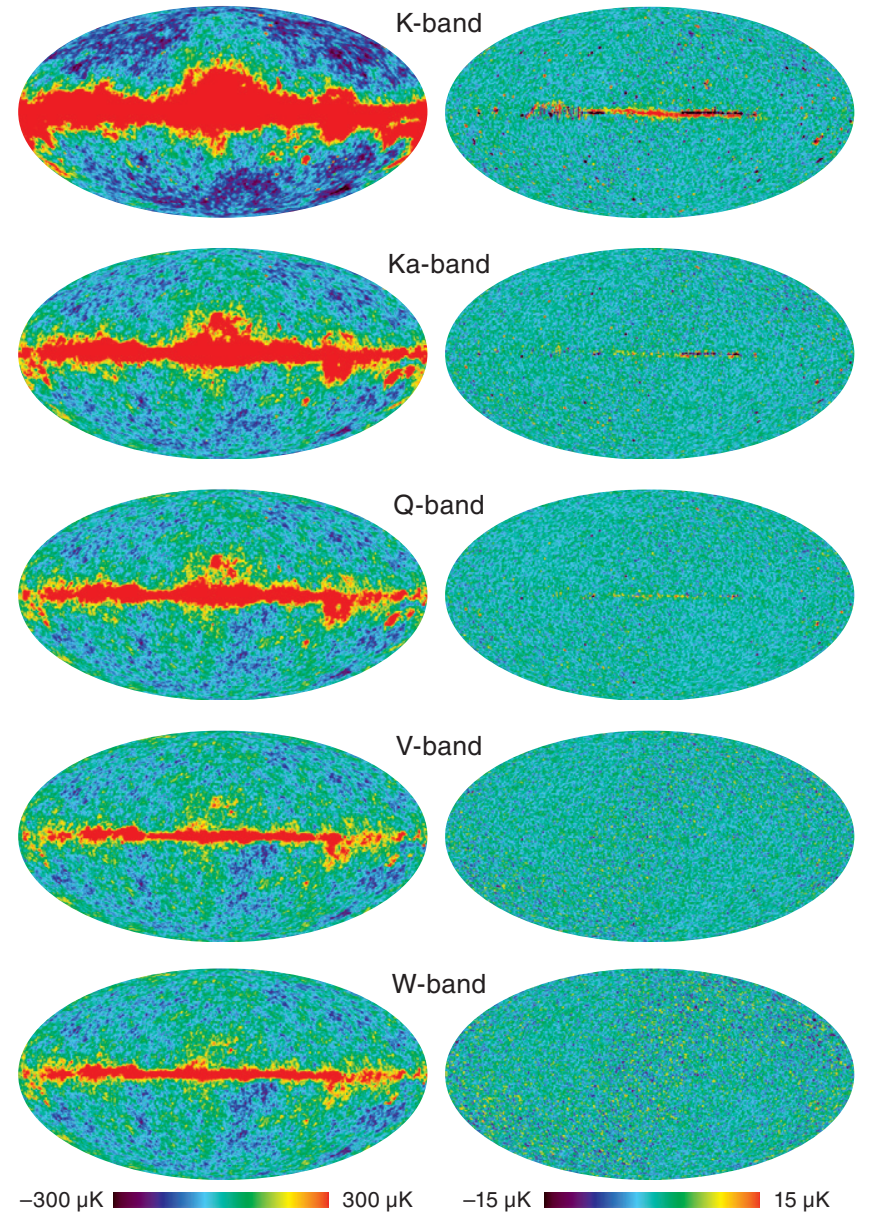

Figure 5. Plots of the Stokes $I$ maps in Galactic coordinates. The left column displays the seven-year average maps, all of which have a common dipole signal removed. The right column displays the difference between the seven-year average maps and the previously published five-year average maps, adjusted to take into account the slightly different dipoles subtracted in the seven-year and five-year analyses and the slightly differing calibrations. All maps have been smoothed with a $1^{\circ}$ FWHM Gaussian kernel. The small Galactic plane signal in the difference maps arises from the difference in calibration $(0.1 \%)$ and beam symmetrization between the five-year and seven-year processing. Note that the temperature scale has been expanded by a factor of 20 for the difference maps.

of the map, they are properly de-weighted when these maps are analyzed using their corresponding $\boldsymbol{\Sigma}^{-1}$ matrices, so useful polarization power spectra may be obtained from these maps. The relatively large amplitudes of these modes limits the utility of using difference plots between the five-year and seven-year map sets to test for consistency. 

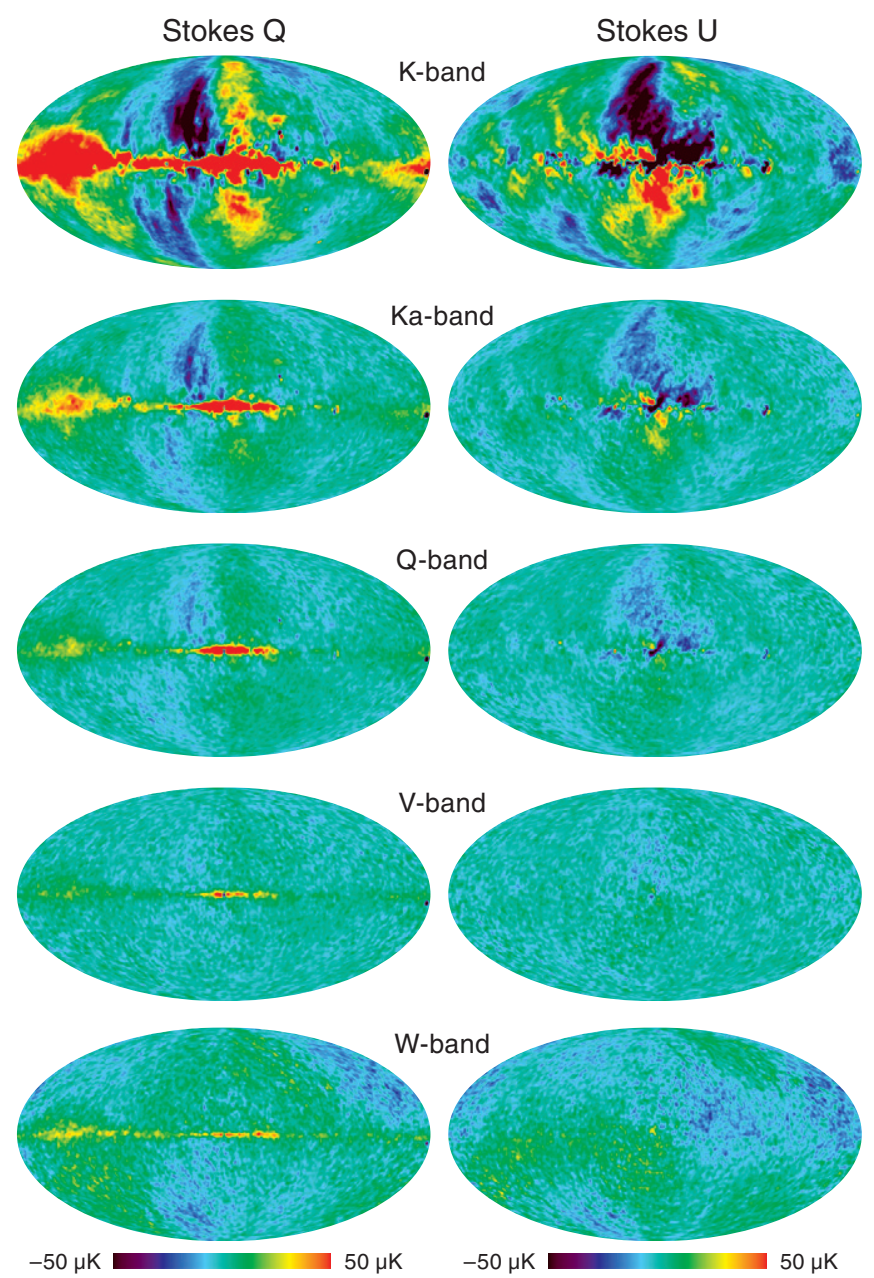

Figure 6. Plots of the seven-year average Stokes $Q$ and $U$ maps in Galactic coordinates. All maps have been smoothed with a $2^{\circ}$ FWHM Gaussian kernel.

\subsubsection{Low- $\ell W$-band Polarization Spectra}

Previous analyses (Hinshaw et al. 2009; Page et al. 2007) exhibited unexplained artifacts in the low- $\ell \mathrm{W}$-band polarization power spectra demonstrating an incomplete understanding of the signal and/or noise properties of these spectra. Specifically, the value of $C_{\ell}^{\mathrm{EE}}$ for $\ell=7$ measured in the $\mathrm{W}$ band was found to be significantly higher than could be accommodated by the best-fit power spectra, given the measurement uncertainty. This result, and several other anomalies, caused these data to be excluded from cosmological analyses. Significant effort has been expended trying to understand these spectra with the goal of eventually allowing their use in cosmological analyses.

A set of null spectra was formed based on the latest uncleaned W-band polarization sky maps to test for year-to-year and DA-to-DA consistency. Polarization cross power spectra were calculated for pairs of maps using the Master algorithm (Hivon et al. 2002) utilizing the full $\boldsymbol{\Sigma}^{-1}$ covariance matrix to weight the input maps. The polarization analysis mask was applied by marginalizing the $\boldsymbol{\Sigma}^{-1}$ over pixels excluded by the mask to minimize foreground contamination. Appropriately weighted null signal combinations of these spectra were formed to determine if any individual years or DAs possessed peculiar characteristics. The uncertainties on the power spectra were evaluated using the Fisher matrix technique (Page et al. 2007) and measured map noise levels. Since the input sky maps contain both signal (mostly of Galactic origin) and noise, an additional
Table 7

Seven-year Spectrum $\chi^{2} W$-band Null Tests

\begin{tabular}{|c|c|c|c|c|}
\hline Data Combinations & $\chi_{\mathrm{EE}}^{2}$ & PTEEE $_{\text {E }}$ & $\chi_{\mathrm{BB}}^{2}$ & $\mathrm{PTE}_{\mathrm{BB}}$ \\
\hline \multicolumn{5}{|c|}{ For DAs W1, W2, W3, and W4 } \\
\hline$\{\mathrm{yr} 1\}-\{\mathrm{y} 2, \mathrm{y} 3, \mathrm{y} 4, \mathrm{y} 5, \mathrm{y} 6, \mathrm{y} 7\}$ & 0.944 & 0.56 & 0.976 & 0.50 \\
\hline$\{\mathrm{yr} 2\}-\{\mathrm{y} 1, \mathrm{y} 3, \mathrm{y} 4, \mathrm{y} 5, \mathrm{y} 6, \mathrm{y} 7\}$ & 0.800 & 0.78 & 0.956 & 0.54 \\
\hline$\{\mathrm{yr} 3\}-\{\mathrm{y} 1, \mathrm{y} 2, \mathrm{y} 4, \mathrm{y} 5, \mathrm{y} 6, \mathrm{y} 7\}$ & 0.934 & 0.57 & 1.166 & 0.24 \\
\hline$\{\mathrm{yr} 4\}-\{\mathrm{y} 1, \mathrm{y} 2, \mathrm{y} 3, \mathrm{y} 5, \mathrm{y} 6, \mathrm{y} 7\}$ & 0.850 & 0.70 & 0.920 & 0.59 \\
\hline$\{\mathrm{yr} 5\}-\{\mathrm{y} 1, \mathrm{y} 2, \mathrm{y} 3, \mathrm{y} 4, \mathrm{y} 6, \mathrm{y} 7\}$ & 0.737 & 0.85 & 1.286 & 0.13 \\
\hline$\{\mathrm{yr} 6\}-\{\mathrm{y} 1, \mathrm{y} 2, \mathrm{y} 3, \mathrm{y} 4, \mathrm{y} 5, \mathrm{y} 7\}$ & 0.769 & 0.82 & 1.101 & 0.32 \\
\hline$\{\mathrm{yr} 7\}-\{\mathrm{y} 1, \mathrm{y} 2, \mathrm{y} 3, \mathrm{y} 4, \mathrm{y} 5, \mathrm{y} 6\}$ & 1.108 & 0.31 & 0.831 & 0.73 \\
\hline$\{\mathrm{yr} 1, \mathrm{yr} 2, \mathrm{y} 3\}-\{\mathrm{y} 4, \mathrm{y} 5, \mathrm{y} 6, \mathrm{y} 7\}$ & 0.608 & 0.96 & 0.98 & 0.49 \\
\hline$\{\mathrm{yr} 1, \mathrm{yr} 3, \mathrm{y} 5, \mathrm{y} 7\}-\{\mathrm{y} 2, \mathrm{y} 4, \mathrm{y} 6\}$ & 0.860 & 0.69 & 1.06 & 0.38 \\
\hline \multicolumn{5}{|c|}{ For seven years of data } \\
\hline$\{\mathrm{W} 1\}-\{\mathrm{W} 2, \mathrm{~W} 3, \mathrm{~W} 4\}$ & 1.195 & 0.21 & 0.982 & 0.49 \\
\hline$\{\mathrm{W} 2\}-\{\mathrm{W} 1, \mathrm{~W} 3, \mathrm{~W} 4\}$ & 0.802 & 0.77 & 0.926 & 0.58 \\
\hline$\{\mathrm{W} 3\}-\{\mathrm{W} 1, \mathrm{~W} 2, \mathrm{~W} 4\}$ & 0.890 & 0.64 & 0.897 & 0.63 \\
\hline$\{\mathrm{W} 4\}-\{\mathrm{W} 1, \mathrm{~W} 2, \mathrm{~W} 3\}$ & 1.163 & 0.24 & 2.061 & 0.0005 \\
\hline$\{\mathrm{W} 1, \mathrm{~W} 2\}-\{\mathrm{W} 3, \mathrm{~W} 4\}$ & 0.867 & 0.68 & 1.361 & 0.09 \\
\hline$\{\mathrm{W} 1, \mathrm{~W} 3\}-\{\mathrm{W} 2, \mathrm{~W} 4\}$ & 0.866 & 0.68 & 1.219 & 0.19 \\
\hline
\end{tabular}

Notes. $\chi^{2}$ tests for various null combinations of low- $\ell$ Master polarization power spectra obtained from uncleaned W-band sky maps. Reduced $\chi^{2}$ values are presented along with the probability to exceed (PTE) values based on 31 degrees of freedom, corresponding to $2 \leqslant \ell \leqslant 32$. Polarization cross power spectra were obtained for each DA year $\times$ DA year combination, then appropriately weighted combinations generated to null any sky signal. Predicted uncertainties were obtained using the standard Fisher matrix formalism incorporating the inverse noise covariance matrices and the measured sky map noise levels. Since individual power spectra estimates do include signals (mostly foreground), the uncertainties include a contribution for the signal $\times$ noise cross term as explained in the text. The only anomalous point occurs when the seven-year W4 data are compared to the seven-year data from the remaining W-band DAs.

term was added to the Fisher matrix noise estimate to account for the signal $\times$ noise cross term. The signal component of this term was estimated using the seven-year average power spectra values for the combined W1, W2, and W3 DAs. This term was only added for multipole/polarization combinations (EE or BB) for which the estimated signal was greater than 0 .

Table 7 shows the result of this analysis. The reduced $\chi^{2}$ combinations in the top panel are evaluated for data combinations of all four W-band DAs that compare individual years to the average of the remaining years. Polarization combinations $\mathrm{EE}$ and $\mathrm{BB}$ were evaluated for multipoles ranging from 2 to 32. Additional combinations were formed to compare the first three years of data to the latter four, and to compare data taken in odd and even numbered years. All combinations resulted in reasonable $\chi^{2}$ and probability to exceed values.

The lower half of the table contains the results of a similar analysis, but in this case combinations were formed to isolate individual DAs. The W4 is singled out with a reduced $\chi^{2}$ value of 1.163 which has only a probability to exceed of only $0.05 \%$. This DA has an unusually large $1 / f$ knee frequency, which makes it particularly susceptible to systematic artifacts. For this reason it is excluded from the analysis below, but continues to be studied in case it might contain clues as to the nature of the low- $\ell$ polarization anomalies seen in the other $W$-band DAs.

Figure 7 displays polarization power spectra for the EE, BB, and EB modes for the first three, five, and seven years of template cleaned individual year maps from the W1, W2, and W3 DAs. These spectra were also obtained using the Master algorithm utilizing the mask-marginalized $\boldsymbol{\Sigma}^{-1}$ covariance matrix sky map weighting. Only cross power spectra are included, so 


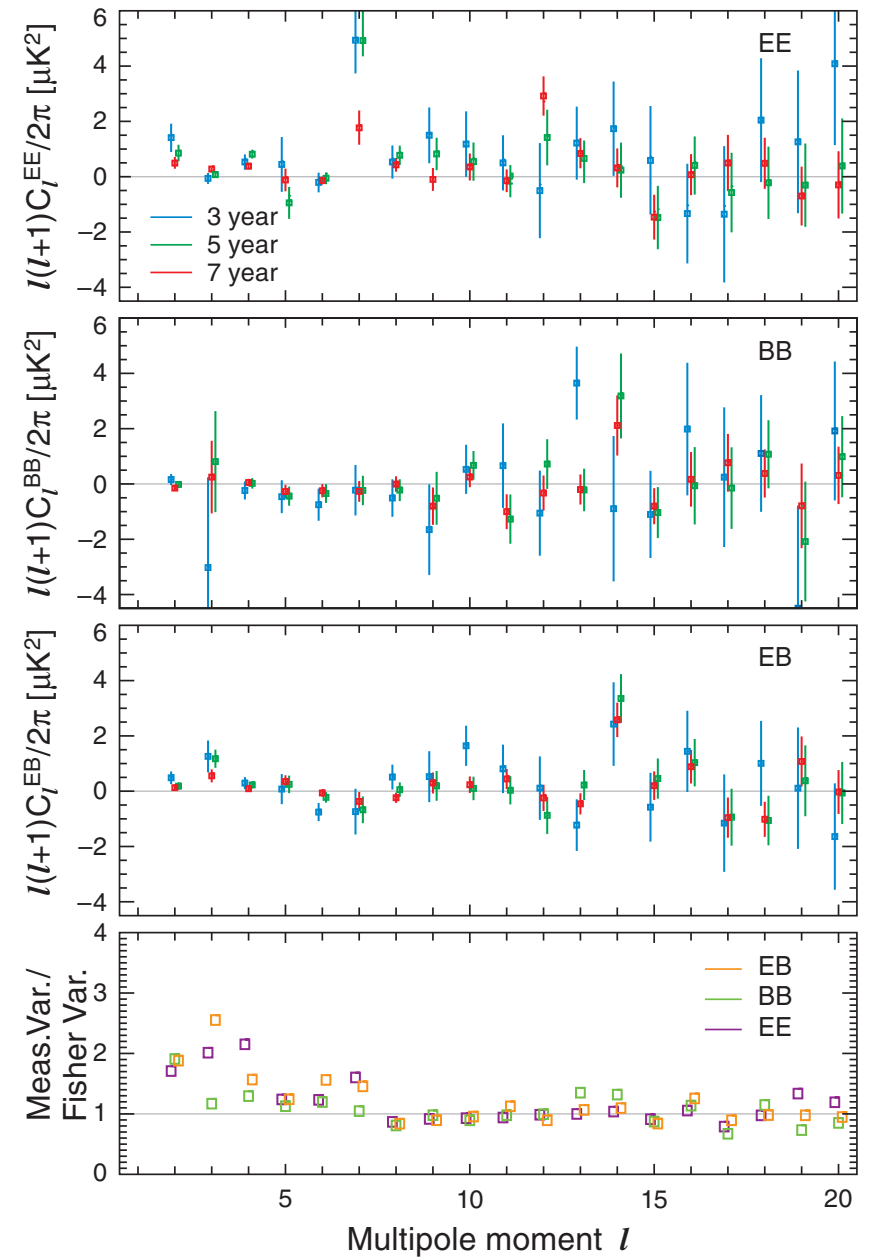

Figure 7. Low- $\ell$ Master polarization cross power spectra from the first three, five, and seven years of data from the $\mathrm{W} 1, \mathrm{~W} 2$, and $\mathrm{W} 3$ foregrounds reduced polarization sky maps (Gold et al. 2011). These three time ranges contain 36, 105 , and 210 individual cross spectra, respectively. The $\ell$ values for the different time ranges have been offset for clarity. The top three panels contain the Master power spectra and error bars based on a Fisher matrix analysis. The bottom panel is the ratio of the measured variance between the individual power spectra estimates (DA year $\times$ DA year) and the variance predicted by the Fisher matrix calculation using the full $\Sigma^{-1}$ inverse noise covariance matrix. Note the good agreement for $\ell \geqslant 8$.

the measurement noise does not bias the measured values. The uncertainties are based on the Fisher matrix technique, and have not had the signal $\times$ noise term added, since any signal remaining in the cleaned maps is expected to be small. (Master algorithm based low- $\ell$ power spectra are not used in any cosmological analysis.)

There is generally good agreement between the values for the three different time ranges. Note that the high value seen in the three- and five-year analyses for $C_{7}^{\mathrm{EE}}$ has fallen significantly with the additional two years of data. In fact, had the data been taken in reverse order (starting with year seven), the $C_{7}^{\mathrm{EE}}$ value would not have been identified as particularly anomalous. However, the $C_{12}^{\mathrm{EE}}$ value has risen in the seven-year combination. To see if these signals might be artifacts of the foreground cleaning, a similar analysis was performed on the uncleaned sky maps and is displayed in Figure 8. By comparing the two figures, it can be seen that the foreground cleaning mainly affects the multipoles $\ell \leqslant 5$ leaving the $\ell=7,12$ multipoles relatively unchanged.
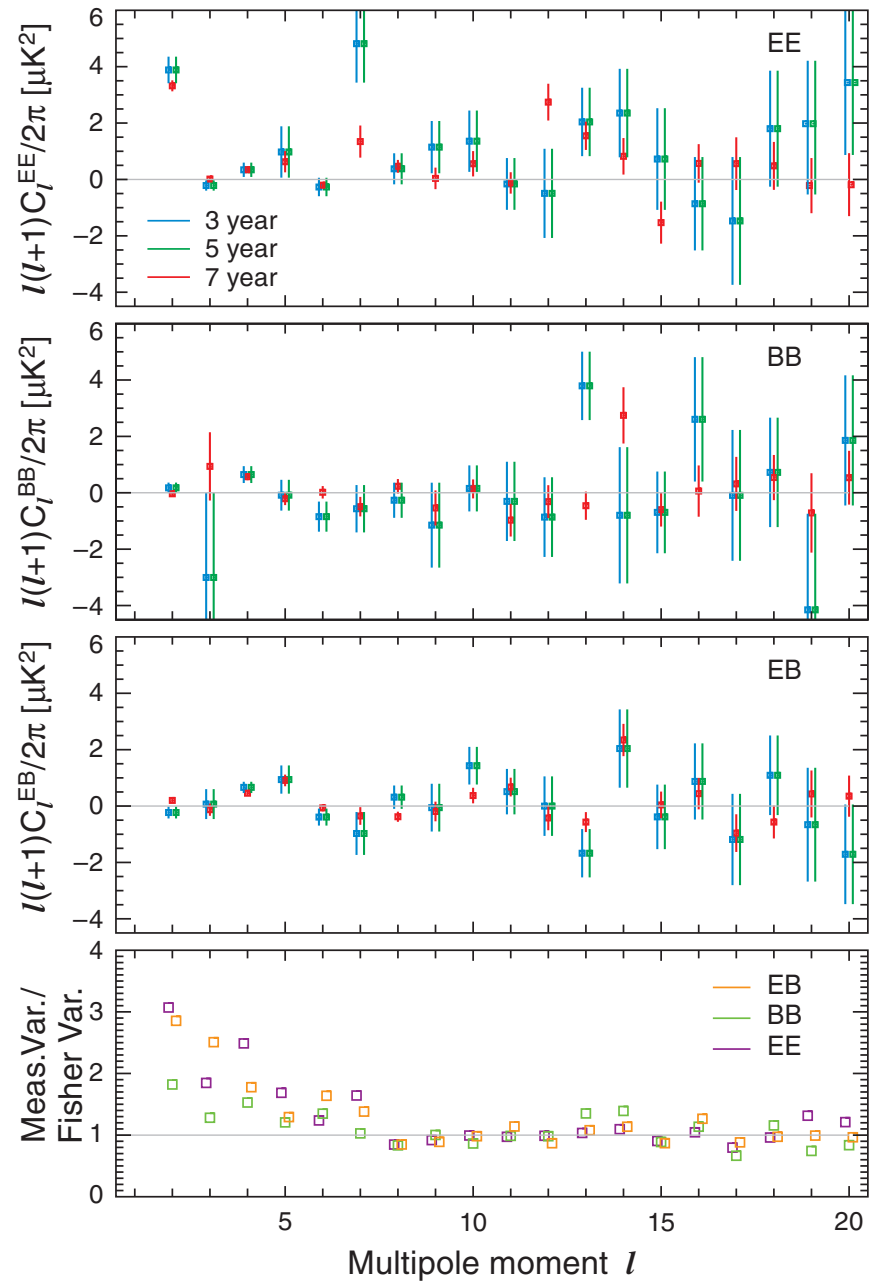

Figure 8. Low- $\ell$ Master polarization cross power spectra from the first three, five, and seven years of data from the W1, W2, and W3 uncleaned sky maps. The $\ell$ values have been offset slightly for the different time ranges for clarity. The bottom panel is the ratio of the measured variance between the individual power spectra estimates (DA year $\times$ DA year) and the variance predicted from a Fisher matrix calculation using the full $\Sigma^{-1}$ inverse noise covariance matrix. Note the good agreement for $\ell \geqslant 8$.

The bottom panel of these figures plots the ratio between the variance measured among the individual cross spectra for each multipole/polarization combination, to that predicted by the Fisher matrix technique. For $\ell \geqslant 8$ there is good agreement between the measured variance and analytical estimate, but the measured variance slowly grows larger with decreasing $\ell$ for $\ell \leqslant 7$ for both the cleaned and uncleaned sky maps. The Fisher matrix noise estimate utilizes the $\boldsymbol{\Sigma}^{-1}$ matrix that describes the noise correlation between the pixels of the Stokes $Q$ and $U$ maps. This matrix incorporates information regarding the sky scanning pattern, through the mapping matrices $\mathbf{M}$ and $\mathbf{M}_{\mathrm{am}}$, and correlations in the radiometer noise, through the use of inverse radiometer noise covariance matrix $\mathbf{N}^{-1}$. This method does not, however, include the effect of any noise correlations that might be introduced through the baseline fitting and calibration procedure. A program was undertaken to determine if the baseline fitting and calibration procedure could be affecting the low- $\ell$ polarization results and is described in the following section. 


\subsubsection{Calibration-induced Spectral Uncertainties}

Uncertainties in the polarization power spectra arising from calibration of the TOD were evaluated using the Fisher information matrix method. New sets of noise matrices were calculated including degrees of freedom associated with the gain and baseline parameters used in the calibration procedure. The values of these two parameters were approximated as piecewise functions, each assumed constant over one hour intervals. The calibration procedure was modeled as a $\chi^{2}$ minimization of the residuals between the TOD and a predicted TOD calculated by applying the gain and baseline values for each time interval to a model signal consisting of CMB anisotropy and a time-dependent dipole due to WMAP's motion about the solar system barycenter. Fisher information matrices were calculated for this model and inverted to form noise matrices. Uncertainties in the recovered low- $\ell$ power spectra were calculated using this method by marginalizing over the values of the gain and baseline parameters. A similar calculation was performed with the gain and baseline terms omitted. The effects of the calibration on the uncertainties in the recovered power spectra were measured by comparing the results of these two calculations.

As expected, the difference in the predicted uncertainties was small for all but the very lowest multipoles. This occurs because the signal from the low- $\ell$ multipoles enters the TOD on the longest timescales, and therefore is most affected by a the calibration procedure which fits the low frequency dipole signal used for calibration. For E-mode polarization the maximum increase in uncertainty in $a_{l m}$ is about $3.5 \%$ occurring for $\ell=3$ and is less than $1 \%$ for other multipoles. For the B-mode $a_{l m}$ uncertainties for $\ell=2,3$ increase by $40 \%$ and $90 \%$, respectively, while the effect on other multipoles is less that $1 \%$. The $\ell=3 \mathrm{~B}$-mode polarization was already known to be very poorly measured by WMAP, since its symmetry, combined with WMAP's geometry and scan pattern, generate extremely long period signals (periods exceeding 10 minutes) in the TOD. The fact that the calculation described above correctly identified this mode supports the validity of the methodology used, but the overall results do no fully explain the excess variance observed in all the $\mathrm{W}$-band $\ell \leqslant 7$ polarization multipoles. The low- $\ell \mathrm{W}$-band polarization data therefore continue to be excluded from cosmological analysis. However, there is no evidence suggesting any compromise of the high- $\ell \mathrm{W}$-band polarization data, so these are now included in evaluation of the high- $\ell$ TE power spectrum.

\subsection{Science Highlights}

The WMAP data remain one of the cornerstone data sets used for testing the cosmological models and the precision measurement of their parameters. Figure 9 displays the binned TT and TE angular power spectra measured from the sevenyear WMAP data (Larson et al. 2011), along with the predicted spectrum for the best-fit minimal six-parameter flat $\Lambda \mathrm{CDM}$ model. The overall agreement is excellent, supporting the validity of this model. Table 8 tabulates the parameter values for this model using WMAP data alone, and in combination with other data sets. Details of the methodology used to determine these values are described in Larson et al. (2011) and Komatsu et al. (2011).

The seven-year WMAP results significantly reduce the uncertainties for numerous cosmological parameters relative to the five-year results. The uncertainties in the densities of bary-
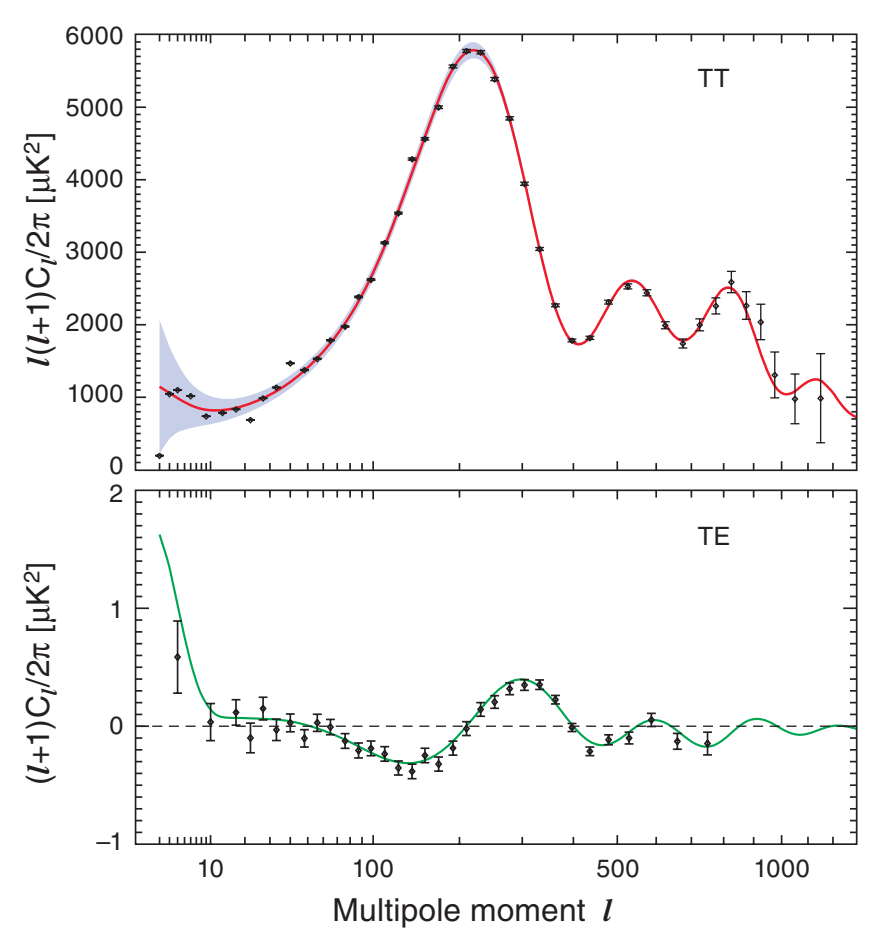

Figure 9. Temperature (TT) and temperature-polarization (TE) power spectra for the seven-year WMAP data set. The solid lines show the predicted spectrum for the best-fit flat $\Lambda C D M$ model. The error bars on the data points represent measurement errors, while the shaded region indicates the uncertainty in the model spectrum arising from cosmic variance. The model parameters are $\Omega_{b} h^{2}$ $=0.02260 \pm 0.00053, \Omega_{c} h^{2}=0.1123 \pm 0.0035, \Omega_{\Lambda}=0.728_{-0.016}^{+0.015}, n_{s}=$ $0.963 \pm 0.012, \tau=0.087 \pm 0.014$, and $\sigma_{8}=0.809 \pm 0.024$.

onic and dark matter are reduced by $10 \%$ and $13 \%$, respectively. When tensor modes are included, the upper bound to their amplitude, determined using WMAP data alone, is nearly $20 \%$ lower. By combining WMAP data with the latest distance measurements from Baryon Acoustic Oscillations (BAO) in the distribution of galaxies (Percival et al. 2010) and Hubble constant measurements (Riess et al. 2009), the spectral index of the power spectrum of primordial curvature perturbations is $n_{s}=0.963 \pm 0.012$, excluding the Harrison-Zel'dovich-Peebles spectrum by more than $3 \sigma$.

The reduced noise obtained by using the seven-year data set yields a better measurement of the third acoustic peak in the temperature power spectrum. This measurement, when combined with external data sets, leads to better determinations of the total mass of neutrinos, $\sum m_{v}$, and the effective number of neutrino species, $N_{\text {eff }}$, as presented in Table 8. Additionally, when augmented by the small scale CMB anisotropy measurements by ACBAR (Reichardt et al. 2009) and QUaD (Brown et al. 2009), this result yields a greater that $3 \sigma$ detection of the primordial Helium abundance, $Y_{\mathrm{He}}=0.326 \pm 0.075$, using CMB data alone. Komatsu et al. (2011) also demonstrate that, with the larger data set, the expected radial and azimuthal polarization patterns around hot and cold peaks in the CMB can now be observed directly in pixel-space by stacking sky map data. In addition, they now detect the Sunyaev-Zel'dovich effect at $\approx 8 \sigma$ at the location of known galaxy clusters, as determined by ROSAT.

Finally, Weiland et al. (2011) have measured the brightness temperature of Jupiter, Saturn, Mars, Uranus, and Neptune, and five fixed calibrations objects, in all five frequency bands, 
Table 8

WMAP Seven-year Cosmological Parameter Summary

\begin{tabular}{|c|c|c|c|}
\hline Description & Symbol & $W M A P$-only & $W M A P+\mathrm{BAO}+H_{0}$ \\
\hline \multicolumn{4}{|c|}{ Parameters for the Standard $\Lambda$ CDM Model ${ }^{\mathrm{a}}$} \\
\hline Age of universe & $t_{0}$ & $13.75 \pm 0.13 \mathrm{Gyr}$ & $13.75 \pm 0.11 \mathrm{Gyr}$ \\
\hline Hubble constant & $H_{0}$ & $71.0 \pm 2.5 \mathrm{~km} \mathrm{~s}^{-1} \mathrm{Mpc}^{-1}$ & $70.4_{-1.4}^{+1.3} \mathrm{~km} \mathrm{~s}^{-1} \mathrm{Mpc}^{-1}$ \\
\hline Baryon density & $\Omega_{b}$ & $0.0449 \pm 0.0028$ & $0.0456 \pm 0.0016$ \\
\hline Physical baryon density & $\Omega_{b} h^{2}$ & $0.02258_{-0.00056}^{+0.00057}$ & $0.02260 \pm 0.00053$ \\
\hline Dark matter density & $\Omega_{c}$ & $0.222 \pm 0.026$ & $0.227 \pm 0.014$ \\
\hline Physical dark matter density & $\Omega_{c} h^{2}$ & $0.1109 \pm 0.0056$ & $0.1123 \pm 0.0035$ \\
\hline Dark energy density & $\Omega_{\Lambda}$ & $0.734 \pm 0.029$ & $0.728_{-0.016}^{+0.015}$ \\
\hline Curvature fluctuation amplitude, $k_{0}=0.002 \mathrm{Mpc}^{-1 \mathrm{~b}}$ & $\Delta_{\mathcal{R}}^{2}$ & $(2.43 \pm 0.11) \times 10^{-9}$ & $\left(2.441_{-0.092}^{+0.088}\right) \times 10^{-9}$ \\
\hline Fluctuation amplitude at $8 h^{-1} \mathrm{Mpc}$ & $\sigma_{8}$ & $0.801 \pm 0.030$ & $0.809 \pm 0.024$ \\
\hline Scalar spectral index & $n_{s}$ & $0.963 \pm 0.014$ & $0.963 \pm 0.012$ \\
\hline Redshift of matter-radiation equality & $z_{\text {eq }}$ & $3196_{-133}^{+134}$ & $3232 \pm 87$ \\
\hline Angular diameter distance to matter-radiation eq. ${ }^{c}$ & $d_{A}\left(z_{\mathrm{eq}}\right)$ & $14281_{-161}^{+158} \mathrm{Mpc}$ & $14238_{-129}^{+128} \mathrm{Mpc}$ \\
\hline Redshift of decoupling & $z_{*}$ & $1090.79_{-0.92}^{+0.94}$ & $1090.89_{-0.69}^{+0.68}$ \\
\hline Age at decoupling & $t_{*}$ & $379164_{-5243}^{+5187} \mathrm{yr}$ & $377730_{-3200}^{+3205} \mathrm{yr}$ \\
\hline Angular diameter distance to decoupling ${ }^{\mathrm{c}, \mathrm{d}}$ & $d_{A}\left(z_{*}\right)$ & $14116_{-163}^{+160} \mathrm{Mpc}$ & $14073_{-130}^{+129} \mathrm{Mpc}$ \\
\hline Sound horizon at decoupling ${ }^{\mathrm{d}}$ & $r_{s}\left(z_{*}\right)$ & $146.6_{-1.6}^{+1.5} \mathrm{Mpc}$ & $146.2 \pm 1.1 \mathrm{Mpc}$ \\
\hline Acoustic scale at decoupling $^{\mathrm{d}}$ & $l_{A}\left(z_{*}\right)$ & $302.44 \pm 0.80$ & $302.40 \pm 0.73$ \\
\hline Reionization optical depth & $\tau$ & $0.088 \pm 0.015$ & $0.087 \pm 0.014$ \\
\hline Redshift of reionization & $z_{\text {reion }}$ & $10.5 \pm 1.2$ & $10.4 \pm 1.2$ \\
\hline \multicolumn{4}{|c|}{ Parameters for Extended Models ${ }^{\mathrm{e}}$} \\
\hline Total density ${ }^{\mathrm{f}}$ & $\Omega_{\text {tot }}$ & $1.080_{-0.071}^{+0.093}$ & $1.0023_{-0.0054}^{+0.0056}$ \\
\hline Equation of state ${ }^{\mathrm{g}}$ & $w$ & $-1.12_{-0.43}^{+0.42}$ & $-0.980 \pm 0.053$ \\
\hline Tensor-to-scalar ratio, $k_{0}=0.002 \mathrm{Mpc}^{-1 \mathrm{~b}, \mathrm{~h}}$ & $r$ & $<0.36(95 \% \mathrm{CL})$ & $<0.24(95 \% \mathrm{CL})$ \\
\hline Running of spectral index, $k_{0}=0.002 \mathrm{Mpc}^{-1 \mathrm{~b}, \mathrm{i}}$ & $d n_{s} / d \ln k$ & $-0.034 \pm 0.026$ & $-0.022 \pm 0.020$ \\
\hline Neutrino density ${ }^{\mathrm{j}}$ & $\Omega_{v} h^{2}$ & $<0.014(95 \% \mathrm{CL})$ & $<0.0062(95 \% \mathrm{CL})$ \\
\hline Neutrino mass ${ }^{\mathrm{j}}$ & $\sum m_{v}$ & $<1.3 \mathrm{eV}(95 \% \mathrm{CL})$ & $<0.58 \mathrm{eV}(95 \% \mathrm{CL})$ \\
\hline Number of light neutrino families ${ }^{\mathrm{k}}$ & $N_{\text {eff }}$ & $>2.7(95 \% \mathrm{CL})$ & $4.34_{-0.88}^{+0.86}$ \\
\hline
\end{tabular}

Notes.

a The parameters reported in the first section assume the six-parameter flat $\Lambda$ CDM model, first using WMAP data only (Larson et al. 2011) and then using $W M A P+\mathrm{BAO}+H_{0}$ data (Komatsu et al. 2011). The $H_{0}$ data consist of a Gaussian prior on the present-day value of the Hubble constant, $H_{0}=74.2 \pm 3.6 \mathrm{~km} \mathrm{~s}^{-1} \mathrm{Mpc}^{-1}$ (Riess et al. 2009), while the BAO priors on the distance ratio $r_{s}\left(z_{d}\right) / D_{\mathrm{V}}(z)$ at $z=0.2,0.3$ are obtained from the Sloan Digital Sky Survey Data Release 7 (Percival et al. 2010). Uncertainties are 68\% CL unless otherwise noted.

${ }^{\mathrm{b}} k=0.002 \mathrm{Mpc}^{-1} \longleftrightarrow l_{\text {eff }} \approx 30$.

c Comoving angular diameter distance.

$\mathrm{d} l_{A}\left(z_{*}\right) \equiv \pi d_{A}\left(z_{*}\right) r_{s}\left(z_{*}\right)^{-1}$.

e The parameters reported in the second section place limits on deviations from the $\Lambda$ CDM model, first using $W M A P$ data only (Larson et al. 2011) and then using $W M A P+\mathrm{BAO}+H_{0}$ data (Komatsu et al. 2011), except as noted otherwise. A complete listing of all parameter values and uncertainties for each of the extended models studied is available on LAMBDA.

${ }^{\mathrm{f}}$ Allows non-zero curvature, $\Omega_{k} \neq 0$.

g Allows $w \neq-1$, but assumes $w$ is constant and $\Omega_{k}=0$. The value in the last column is obtained from a combination of $W M A P+\mathrm{BAO}$ data and luminosity distance information obtained from the "constitution" SNe data set (Hicken et al. 2009) using the methodology described in Komatsu et al. (2011).

${ }^{\mathrm{h}}$ Allows tensor modes but no running in the scalar spectral index.

${ }^{\mathrm{i}}$ Allows running in the scalar spectral index but no tensor modes.

j Allows a massive neutrino component, $\Omega_{v} \neq 0$.

${ }^{\mathrm{k}}$ Allows $N_{\mathrm{eff}}$ number of relativistic species, with the prior $0<N_{\text {eff }}<10$.

allowing their use as millimeter-wave celestial calibration sources traceable to WMAP's precise calibration.

\section{SUMMARY}

The WMAP observatory has successfully completed seven years of observations with no significant performance degradation. A full set of sky maps for the seven-year data span has been generated and is available for analyses by the astrophysical community. These maps were generated with an updated masking procedure that simplifies the map-making procedure and allows creation of a single full-sky noise correlation matrix describing the noise correlation over the entire sky for the reduced resolution sky maps. The understanding of the beam profiles and resulting window functions has been improved with the additional beam profile information obtained by more observations 
of Jupiter. The planetary observations, combined with the precise absolute calibration of WMAP, were used to measure the brightness temperature of Mars, Jupiter, Saturn, Uranus, and Neptune for use as calibration sources.

Finally, the additional data and a better understanding of the instrument's characteristics have resulted in tighter constraints on the value of parameters of cosmological models.

WMAP is funded by the Science Mission Directorate Office at NASA Headquarters. We acknowledge the use of the HEALPix (Gorski et al. 2005) software package. The numerous data products described in this document are available from the Legacy Archive for Microwave Background Data Analysis (LAMBDA): http://lambda.gsfc.nasa.gov.

\section{REFERENCES}

Barrett, R., et al. 1994, Templates for the Solution of Linear Systems: Building Blocks for Iterative Methods (2nd ed.; Philadelphia, PA: SIAM)

Bennett, C. L., et al. 2003a, ApJS, 148, 1

Bennett, C. L., et al. 2003b, ApJ, 583, 1

Bennett, C., et al. 2011, ApJS, 192, 17

Brown, M. L., et al. 2009, ApJ, 705, 978
Gold, B., et al. 2011, ApJS, 192, 15

Gorski, K. M., Hivon, E., Banday, A. J., Wandelt, B. D., Hansen, F. K., Reinecke, M., \& Bartlemann, M. 2005, ApJ, 622, 759

Hicken, M., Wood-Vasey, W. M., Blondin, S., Challis, P., Jha, S., Kelly, P. L., Rest, A., \& Kirshner, R. P. 2009, ApJ, 700, 1097

Hill, R. S., et al. 2009, ApJS, 180, 246

Hinshaw, G., et al. 2003, ApJS, 148, 63

Hinshaw, G., et al. 2009, ApJS, 180, 225

Hivon, E., Górski, K. M., Netterfield, C. B., Crill, B. P., Prunet, S., \& Hansen, F. 2002, ApJ, 567, 2

Jarosik, N., et al. 2003, ApJS, 145, 413

Jarosik, N., et al. 2007, ApJS, 170, 263

Komatsu, E., et al. 2011, ApJS, 192, 18

Larson, D., et al. 2011, ApJS, 192, 16

Limon, M., et al. 2010, in Wilkinson Microwave Anisotropy Probe (WMAP): Seven Year Explanatory Supplement, (Greenbelt, MD: NASA GSFC), http:// lambda.gsfc.nasa.gov/product/map/dr4/pub_papers/sevenyear/supplement/ WMAP_supplement.pdf

Mather, J. C., Fixsen, D. J., Shafer, R. A., Mosier, C., \& Wilkinson, D. T. 1999, ApJ, 512, 511

Page, L., et al. 2003a, ApJS, 148, 39

Page, L., et al. 2003b, ApJ, 585, 566

Page, L., et al. 2007, ApJS, 170, 335

Percival, W. J., et al. 2010, MNRAS, 401, 2148

Reichardt, C. L., et al. 2009, ApJ, 694, 1200

Riess, A. G., et al. 2009, ApJ, 699, 539

Weiland, J., et al. 2011, ApJS, 192, 19 